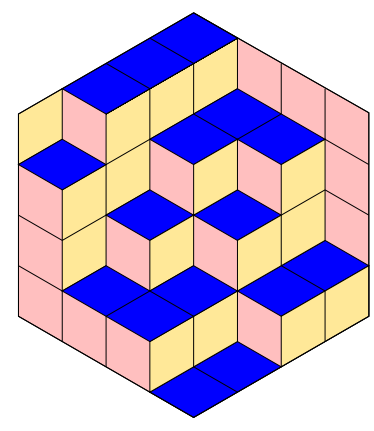

ALGEBRAIC COMBINATORICS

Velleda Baldoni, Nicole Berline, Jesús A. De Loera, Matthias Köppe \& Michèle Vergne

Three Ehrhart quasi-polynomials

Volume 2, issue 3 (2019), p. 379-416.

<http://alco.centre-mersenne.org/item/ALCO_2019__2_3_379_0>

(C) The journal and the authors, 2019.

Some rights reserved.

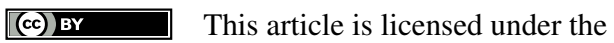

CREATIVE COMmons ATtRIBUtion 4.0 InTERnATIONAL LiCEnSE.

http://creativecommons.org/licenses/by/4.0/

Access to articles published by the journal Algebraic Combinatorics on the website http://alco.centre-mersenne.org/ implies agreement with the Terms of Use (http://alco.centre-mersenne.org/legal/).

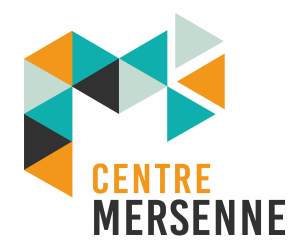

Algebraic Combinatorics is member of the Centre Mersenne for Open Scientific Publishing www.centre-mersenne.org 


\title{
Three Ehrhart quasi-polynomials
}

\author{
Velleda Baldoni, Nicole Berline, Jesús A. De Loera, \\ Matthias Köppe \& Michèle Vergne
}

Abstract Let $\mathfrak{p}(b) \subset \mathbb{R}^{d}$ be a semi-rational parametric polytope, where $b=\left(b_{j}\right) \in \mathbb{R}^{N}$ is a real multi-parameter. We study intermediate sums of polynomial functions $h(x)$ on $\mathfrak{p}(b)$,

$$
S^{L}(\mathfrak{p}(b), h)=\sum_{y} \int_{\mathfrak{p}(b) \cap(y+L)} h(x) \mathrm{d} x,
$$

where we integrate over the intersections of $\mathfrak{p}(b)$ with the subspaces parallel to a fixed rational subspace $L$ through all lattice points, and sum the integrals. The purely discrete sum is of course a particular case $(L=0)$, so $S^{0}(\mathfrak{p}(b), 1)$ counts the integer points in the parametric polytopes.

The chambers are the open conical subsets of $\mathbb{R}^{N}$ such that the shape of $\mathfrak{p}(b)$ does not change when $b$ runs over a chamber. We first prove that on every chamber of $\mathbb{R}^{N}, S^{L}(\mathfrak{p}(b), h)$ is given by a quasi-polynomial function of $b \in \mathbb{R}^{N}$. A key point of our paper is an analysis of the interplay between two notions of degree on quasi-polynomials: the usual polynomial degree and a filtration, called the local degree.

Then, for a fixed $k \leqslant d$, we consider a particular linear combination of such intermediate weighted sums, which was introduced by Barvinok in order to compute efficiently the $k+1$ highest coefficients of the Ehrhart quasi-polynomial which gives the number of points of a dilated rational polytope. Thus, for each chamber, we obtain a quasi-polynomial function of $b$, which we call Barvinok's patched quasi-polynomial (at codimension level $k$ ).

Finally, for each chamber, we introduce a new quasi-polynomial function of $b$, the coneby-cone patched quasi-polynomial (at codimension level $k$ ), defined in a refined way by linear combinations of intermediate generating functions for the cones at vertices of $\mathfrak{p}(b)$.

We prove that both patched quasi-polynomials agree with the discrete weighted sum $b \mapsto$ $S^{\{0\}}(\mathfrak{p}(b), h)$ in the terms corresponding to the $k+1$ highest polynomial degrees.

\section{INTRODUCTION}

In this article, a parametric semi-rational polytope $\mathfrak{p}(b) \subset \mathbb{R}^{d}$ is defined by inequalities:

$$
\mathfrak{p}(b)=\left\{x \in \mathbb{R}^{d}:\left\langle\alpha_{j}, x\right\rangle \leqslant b_{j}, j=1, \ldots, N\right\}
$$

where $\alpha_{1}, \alpha_{2}, \ldots, \alpha_{N}$ are fixed linear forms with integer coefficients (the case of rational $\alpha_{j}$ can be treated by rescaling $\alpha_{j}$ and $\left.b\right)$ and the parameter $b=\left(b_{1}, b_{2}, \ldots, b_{N}\right)$ varies in $\mathbb{R}^{N}$.

The shape of the polytope $\mathfrak{p}(b)$ varies when the parameter $b$ varies (see Figure 1 ). Chambers $\tau \subset \mathbb{R}^{N}$ are open convex polyhedral cones such that the shape of $\mathfrak{p}(b)$ does

Manuscript received 27th March 2018, revised 25th October 2018, accepted 1st November 2018. KEYWORDS. Ehrhart polynomials, generating functions, Barvinok's algorithm, parametric polytopes. 

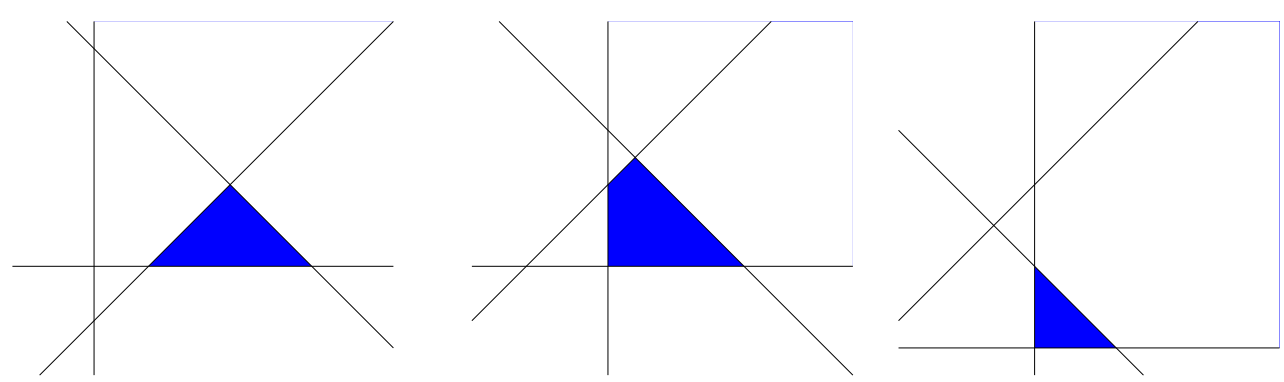

Figure 1. The parametric polytope $\mathfrak{p}(b)$ from Example 2.8, for $b$ in various chambers.

not change when $b$ runs over $\tau$ (see Definition 2.12). We consider weighted integrals and sums, where the weight is a polynomial function $h(x)$ of degree $m$ on $\mathbb{R}^{d}$.

$$
I(\mathfrak{p}(b), h)=\int_{\mathfrak{p}(b)} h(x) \mathrm{d} x, \quad S(\mathfrak{p}(b), h)=\sum_{x \in \mathfrak{p}(b) \cap \mathbb{Z}^{d}} h(x) .
$$

When the weight is the constant 1 , then $I(\mathfrak{p}(b), 1)$ is the volume of $\mathfrak{p}(b)$, while $S(\mathfrak{p}(b), 1)$ is the number of integral points in $\mathfrak{p}(b)$.

As introduced by Barvinok, we also study intermediate sums associated to a rational subspace $L$ :

$$
S^{L}(\mathfrak{p}(b), h)=\sum_{y} \int_{\mathfrak{p}(b) \cap(y+L)} h(x) \mathrm{d} x .
$$

Here, we integrate over the intersections of $\mathfrak{p}(b)$ with the subspaces parallel to a fixed rational subspace $L$ through all lattice points, and sum the integrals.

The unweighted case $(h=1)$, the study of the counting function $S(\mathfrak{p}(b), 1)$ is, of course, very important in algebraic combinatorics. Polytopes depending on multiple parameters have appeared, for example, in the celebrated Knutson-Tao honeycomb model [15]. Also the classical vector partition functions [12] appear as a special case. However, a large part of the literature has focused on the case of one-parameter families of dilations of a single polytope (see our discussion on Ehrhart theory in Section 1.1 below), with few exceptions $[9,14,16]$. Indeed [14] was part of our motivation to consider the case of a real multi-parameter and not just one-parameter dilations as in our previous articles on the subject. Our interest in the general problem $S(\mathfrak{p}(b), h)$ is motivated in part by the important applications in compiler optimization and automatic code parallelization, in which multiple parameters arise naturally (see [13, 20, 21] and the references within). For a broader context of analytic combinatorics, we refer to $[19]$.

The relations between the two functions $I(\mathfrak{p}(b), h)$ and $S(\mathfrak{p}(b), h)$ of the parameter vector $b$ have been the central theme of several works. In this article, we (hope to) add a contribution to these questions.

We introduce the new notion of local degree, which we believe is important. A function $b \mapsto f(b)$ of the real multi-parameter $b$ is of local degree (at most) $\ell$ if it can be expressed as a linear combination of products of a number less or equal to $\ell$ of step-linear forms of $b$ and linear forms of $b$ (see Definition 2.20 below and Figure 3, left). If the number of linear forms is less than or equal to $q$, we say that $f$ is of polynomial degree (at most) $q$. 
The present article is the culmination of a study based on $[4,5]$. These two articles were devoted to the properties of intermediate generating functions only for polyhedral cones. Here, using the Brianchon-Gram set-theoretic decomposition of a polytope as a signed sum of its supporting cones, we study the function $b \mapsto S^{L}(\mathfrak{p}(b), h)$.

We show first that, on each chamber, the function $b \mapsto S^{L}(\mathfrak{p}(b), h)$ is of local degree at most $d+m$. In particular its term of polynomial degree 0 is expressed as a linear combination of at most $d+m$ step-linear functions of $b$.

Then we study the terms of highest polynomial degree of $S(\mathfrak{p}(b), h)$ on each chamber.

Given a fixed integer $k \leqslant d$ we construct two quasi-polynomials, Barvinok's patched quasi-polynomial (at level $k$ ) and the cone-by-cone patched quasi-polynomial (at level $k$ ). The two constructions use linear combinations of intermediate sums associated to rational subspaces $L$ of codimension less or equal to $k$. The first one is due to Barvinok [7]. We give a more streamlined proof of Barvinok's Theorem 1.3 in [7] and a more explicit formula for it when $\mathfrak{p}(b)$ is a simplex. The cone-by-cone patched quasi-polynomial is a new construction. We prove that both patched quasipolynomials agree with the discrete weighted sum $b \mapsto S(\mathfrak{p}(b), h)$ in the terms corresponding to the $k+1$ highest polynomial degrees $d+m, d+m-1, \ldots, d+m-k$.

We now give more details on the content of this article.

1.1. Weighted EHRHART QUASI-POLYNOMIALS AND INTERMEDIATE SUMS. When a rational parameter vector $b$ is fixed, then the polytope $\mathfrak{p}=\mathfrak{p}(b)$ is a rational polytope. If we dilate it by a non-negative number $t$, the function $t \mapsto S(t \mathfrak{p}, h)$ is a quasipolynomial function of $t$, i.e., it takes the form

$$
S(t \mathfrak{p}, h)=E(t)=\sum_{j=0}^{d+m} E_{j}(t) t^{j},
$$

where the coefficients $E_{j}(t)$ are periodic functions of $t$, rather than constants. It is called the weighted Ehrhart quasi-polynomial of $\mathfrak{p}$. In traditional Ehrhart theory, only non-negative integer dilation factors $t$ are considered, and so a coefficient function with period $q \in \mathbb{Z}_{>0}$ can be given as a list of $q$ values, one for each residue class modulo $q$. However, the approach to computing Ehrhart quasi-polynomials via generating functions of parametric polyhedra $[16,20,21]$, which we follow in the present paper, leads to a natural, shorter representation of the coefficient functions as closed-form formulas (so-called step-polynomials) of the dilation parameter $t$, using the "fractional part" function. These closed-form formulas are naturally valid for arbitrary non-negative real dilation parameters $t$, as well as any real (not just rational) parameter $b$. This fact was implicit in the computational works following this method [20, 21], and was made explicit in [16]. The resulting real Ehrhart theory has recently caught the interest of other authors [14, 17]; see also [5].

The highest "expected" degree term of the weighted Ehrhart quasi-polynomial is $I(\mathfrak{p}, h) t^{d+m}$, if $h(x)$ is homogeneous of degree $m$; of course, this term may vanish, as the example $\mathfrak{p}=[-1,1], h(x)=x$ illustrates. For a study of the coefficients of degree $d+m, d+m-1, \ldots, d+m-k$ of the quasi-polynomial $S(t \mathfrak{p}, h)$, a key tool introduced by Barvinok (in [7], for the unweighted case $h=1$ ) is the intermediate weighted sum $S^{L}(\mathfrak{p}, h)$, where $L$ is a rational subspace of $V=\mathbb{R}^{d}$ :

$$
S^{L}(\mathfrak{p}, h)=\sum_{y} \int_{\mathfrak{p} \cap(y+L)} h(x) \mathrm{d} x,
$$

where the summation variable $y$ runs over the projected lattice in $V / L$. The polytope $\mathfrak{p}$ is sliced by subspaces parallel to $L$ through lattice points and the integrals of $h$ over 


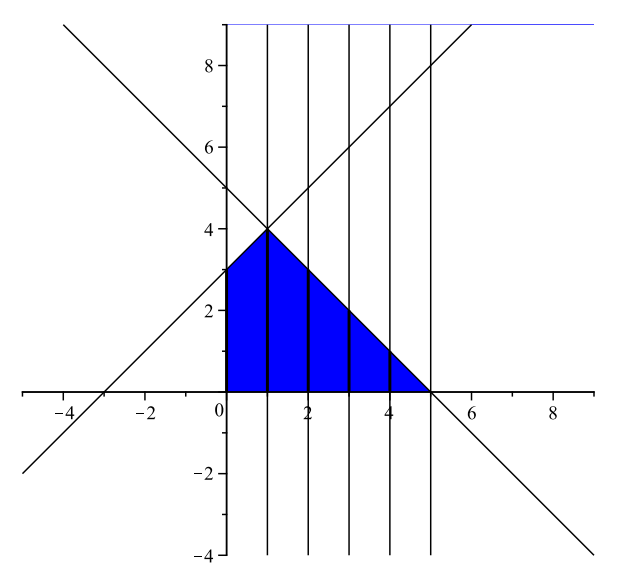

FIGURE 2. Intermediate sum over a polytope $\mathfrak{p}$ (blue). We sum the integrals over the slices of $p$ parallel to $L$ going through lattice points (vertical lines).
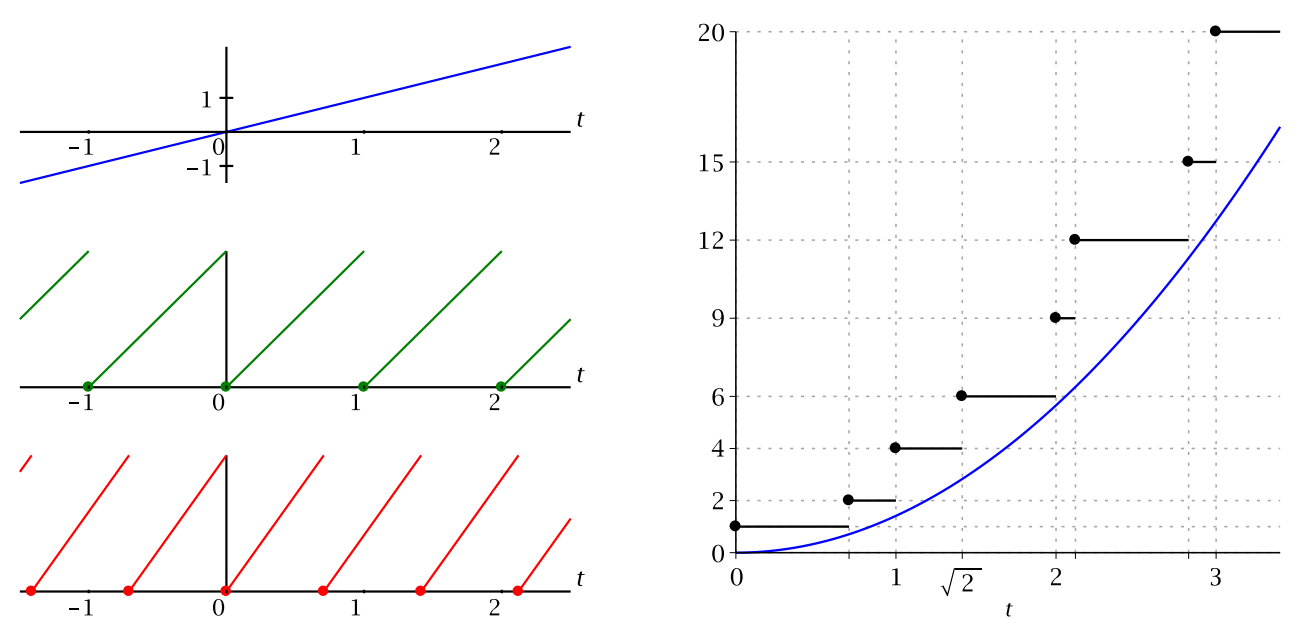

Figure 3. Example 1.4. Left. The linear function $t$ (top, blue), the rational step-linear function $\{t\}$ (center, green), and the irrational step-linear function $\{\sqrt{2} t\}$ (bottom, red). Right. The number of lattice points $($ black) of the rectangle $[0, t] \times[0, t \sqrt{2}]$ is a function in the algebra generated by these three functions; the area (blue) is a polynomial function.

the slices are added (see Figure 2). When $L=V, S^{L}(\mathfrak{p}, h)$ is just the integral $I(\mathfrak{p}, h)$, while for $L=\{0\}$, we recover the discrete sum $S(\mathfrak{p}, h)$. In the present study, we generalize Barvinok's ideas in several ways, building on our previous work in $[3,4,5]$.

1.2. Real multi-Parameter QUaSi-POlynomials and their Degrees. To describe our contributions, let us first define our notion of (real, multi-parameter) quasipolynomials on $\mathbb{R}^{N}$ and notions of degree, which are crucial for our paper.

First we define (rational) step-polynomials. For $t \in \mathbb{R}$, we denote by $\{t\} \in[0,1[$ the fractional part of $t$. Thus $t \mapsto\{t\}$ is a function on $\mathbb{R} / \mathbb{Z}$. Let $\eta=\left(\eta_{1}, \eta_{2}, \ldots, \eta_{N}\right) \in \mathbb{Q}^{N}$, 
which we consider as a linear form on $\mathbb{R}^{N}$. We say that the function $b \mapsto\{\langle\eta, b\rangle\}$ is a (rational) step-polynomial function of (step) degree (at most) one (or a (rational) step-linear function). If all $\eta_{i}$ have the same denominator $q$, this is a function of $b \in \mathbb{R}^{N} / q \mathbb{Z}^{N}$. We define $\mathcal{Q}\left(\mathbb{R}^{N}\right)$ to be the algebra of functions on $\mathbb{R}^{N}$ generated by the functions $b \mapsto\{\langle\eta, b\rangle\}$. An element of $\mathcal{Q}\left(\mathbb{R}^{N}\right)$ is called a (rational) step-polynomial on $\mathbb{R}^{N}$. The space $\mathcal{Q}\left(\mathbb{R}^{N}\right)$ has an obvious filtration, where $\mathcal{Q}_{[\leqslant k]}\left(\mathbb{R}^{N}\right)$ is the linear span of $k$ or fewer products of functions $b \mapsto\{\langle\eta, b\rangle\}$. The elements of $\mathcal{Q}_{[\leqslant k]}\left(\mathbb{R}^{N}\right)$ are said to be (rational) step-polynomials of (step) degree (at most) $k$.

Next, we define $\mathcal{Q P}\left(\mathbb{R}^{N}\right)$ to be the algebra of functions on $\mathbb{R}^{N}$ generated by (rational) step-polynomials and ordinary polynomial functions of $b$. Elements of $\mathcal{Q P}\left(\mathbb{R}^{N}\right)$ are called quasi-polynomials on $\mathbb{R}^{N}$ and take the form

$$
E(b)=\sum_{\substack{j=\left(j_{1}, \ldots, j_{N}\right) \in \mathbb{Z}_{\geqslant 0}^{N} \\|j|=j_{1}+\cdots+j_{N} \leqslant d+m}} E_{j}(b) b^{j},
$$

using multi-index notation for the monomials $b^{j}=b^{j_{1}} \cdots b^{j_{N}}$. Here the $E_{j}(b)$ are step-polynomials.

This definition of quasi-polynomials on $\mathbb{R}^{N}$ is a natural generalization of the notion of quasi-polynomial function on the lattice $\mathbb{Z}^{N}$, which is more familiar in Ehrhart theory and the theory of vector partition functions. Describing quasi-polynomials in this form, using step-polynomials as its coefficient functions, has been implicit in the computational works using the method of parametric generating functions [20, 21]. The extension to real (rather than integer or rational) multi-parameters $b$ appeared in $[16]$.

The algebra $\mathcal{Q P}\left(\mathbb{R}^{N}\right)$ inherits a grading from the degree of polynomials, which we call the polynomial degree. This is the notion of degree that has been used throughout the literature on Ehrhart theory.

Crucial to our study will be the interplay of the polynomial degree with another notion of degree, first introduced in our paper [4]. The algebra $\mathcal{Q P}\left(\mathbb{R}^{N}\right)$ also has a filtration, which we call the local degree. It combines the polynomial degree and the filtration according to step degrees on step-polynomials. For instance, $b \mapsto b_{1} b_{2}^{2}\left\{b_{1}+b_{3}\right\}$ has polynomial degree 3 , step degree 1 , and local degree 4 . This terminology of local degree comes from the fact that on each local region $n<b_{1}+b_{3}<n+1, n \in \mathbb{Z}$, this function coincides with a polynomial function of $b$ of degree 4 .

1.3. First CONTRibution: Intermediate REAL MUlti-PARAmeter Ehrhart QUASI-POLYNOMIALS AND THEIR DEGREE STRUCTURE. Let $L$ be a rational subspace of $V$. We show that $b \mapsto S^{L}(\mathfrak{p}(b), h)$ is given by a quasi-polynomial formula when the real multi-parameter $b$ varies in a chamber. This generalizes results in the literature in various ways.

(1) It extends from the case of discrete sums $(L=\{0\})$ as it appears in Ehrhart theory and the theory of vector partition functions [12] to the general case of intermediate sums.

(2) It generalizes these works also to the real multi-parameter case.

(3) It analyzes the degree structure, i.e., the interplay of local degree and polynomial degree. This is crucial for our second contribution, relating the terms of highest polynomial degree in $b$ of $S(\mathfrak{p}(b), h)$ to those of certain linear combinations of intermediate sums.

Our theorem is the following (see Theorem 2.26 for a more detailed statement). 

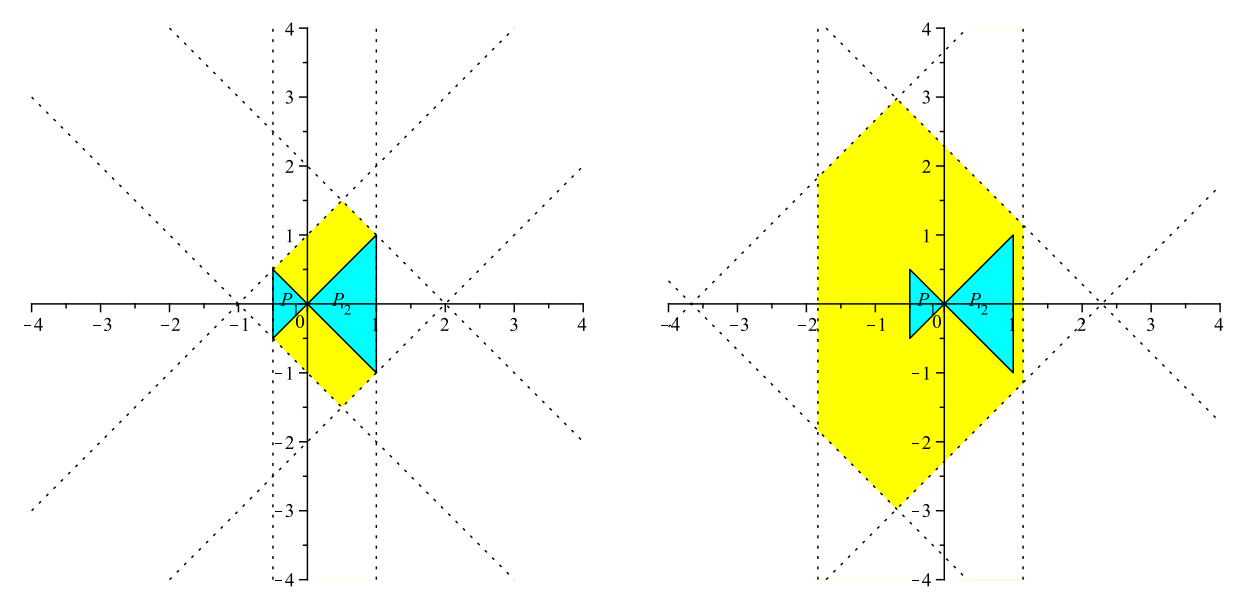

FiguRE 4. Minkowski linear system, Example 1.3. Left, $\mathfrak{p}_{1}+\mathfrak{p}_{2}$. Right, $\frac{11}{3} \mathfrak{p}_{1}+\frac{8}{7} \mathfrak{p}_{2}$.

TheOrem 1.1. Assume that the weight $h(x)$ is homogeneous of degree $m$. When the real multi-parameter $b$ varies in the closure of a chamber, the function $b \mapsto S^{L}(\mathfrak{p}(b), h)$ is given by a quasi-polynomial function of $b$ of local degree equal to $d+m$.

In particular, the terms of highest polynomial degree in $b$ of the function $S^{L}(\mathfrak{p}(b), h)$ form the homogeneous polynomial of degree $d+m$ given by the integral $I(\mathfrak{p}(b), h)$, while the term of polynomial degree 0 (the "constant term") is a step-polynomial of (step) degree at most $d+m$.

EXAMPLE 1.2. The simplest example is $V=\mathbb{R}$ with $\left\langle\alpha_{1}, x\right\rangle=x$ and $\left\langle\alpha_{2}, x\right\rangle=-x$. Thus $\mathfrak{p}(b)=\left\{x \in \mathbb{R}: x \leqslant b_{1},-x \leqslant b_{2}\right\}$. If $b_{1}+b_{2} \geqslant 0$, the polytope $\mathfrak{p}(b)$ is the interval $\left[-b_{2}, b_{1}\right]$. If $L=V$, then $S^{V}(\mathfrak{p}(b), 1)=b_{1}+b_{2}$, while for $L=\{0\}$, $S^{\{0\}}(\mathfrak{p}(b), 1)=b_{1}+b_{2}-\left\{b_{1}\right\}-\left\{b_{2}\right\}+1$. These two functions have local degree 1 with respect to $\left(b_{1}, b_{2}\right)$.

The family $\mathfrak{p}(b)$ is the family of polytopes obtained from a fixed simple rational polytope $\mathfrak{p}$ by moving each facet parallel to itself in all possible ways. We can consider smaller families of polytopes with parallel faces. For example, as in classical Ehrhart theory, we can dilate $\mathfrak{p}$ to obtain $t \mathfrak{p}$ for $t \in \mathbb{R}_{\geqslant 0}$, or more generally we can consider Minkowski linear systems $t_{1} \mathfrak{p}_{1}+t_{2} \mathfrak{p}_{2}+\cdots+t_{q} \mathfrak{p}_{q}$. By specializing our quasi-polynomial formulas, we obtain formulas for $S^{L}(t \mathfrak{p}, h)$ and $S^{L}\left(t_{1} \mathfrak{p}_{1}+t_{2} \mathfrak{p}_{2}+\cdots+t_{q} \mathfrak{p}_{q}, h\right)$.

EXAMPLE 1.3. We consider the Minkowski linear system generated by two triangles $\mathfrak{p}_{1}=\left((0,0),\left(-\frac{1}{2}, \frac{1}{2}\right),\left(-\frac{1}{2},-\frac{1}{2}\right)\right)$ and $\mathfrak{p}_{2}=((0,0),(1,-1),(1,1))$; see Figure 4. Then $t_{1} \mathfrak{p}_{1}+\mathfrak{t}_{2} \mathfrak{p}_{2}$ is the hexagon $\mathfrak{p}\left(\alpha, t_{1} b^{1}+t_{2} b^{2}\right)$, with

$$
\begin{aligned}
\alpha & =\left(x_{1}, x_{1}+x_{2},-x_{1}+x_{2},-x_{1},-x_{1}-x_{2}, x_{1}-x_{2}\right), \\
b^{1} & =\left(0,0,1, \frac{1}{2}, 1,0\right), \\
b^{2} & =(1,2,0,0,0,2) .
\end{aligned}
$$

Its vertices are $\left[t_{2}, t_{2}\right],\left[-\frac{1}{2} t_{1}+t_{2}, \frac{1}{2} t_{1}+t_{2}\right],\left[-\frac{1}{2} t_{1}, \frac{1}{2} t_{1}\right],\left[-\frac{1}{2} t_{1},-\frac{1}{2} t_{1}\right],\left[-\frac{1}{2} t_{1}+t_{2}\right.$, $\left.-\frac{1}{2} t_{1}-t_{2}\right],\left[t_{2},-t_{2}\right]$ 
(1) The volume of $t_{1} \mathfrak{p}_{1}+\mathfrak{t}_{2} \mathfrak{p}_{2}$ is

$$
E_{[2]}\left(t_{1}, t_{2}\right)=\frac{1}{4} t_{1}^{2}+2 t_{1} t_{2}+t_{2}^{2} .
$$

(2) The number $S\left(t_{1}, t_{2}\right)$ of lattice points in $t_{1} \mathfrak{p}_{1}+\mathfrak{t}_{2} \mathfrak{p}_{2}$ is

$$
S\left(t_{1}, t_{2}\right)=E_{[2]}\left(t_{1}, t_{2}\right)+E_{[1]}\left(t_{1}, t_{2}\right)+E_{[0]}\left(t_{1}, t_{2}\right)
$$

where $E_{[1]}\left(t_{1}, t_{2}\right)$ is of polynomial degree 1 and $E_{[0]}\left(t_{1}, t_{2}\right)$ is of polynomial degree 0 , which are given by

$$
E_{[1]}\left(t_{1}, t_{2}\right)=\left(1-\left\{\frac{t_{1}}{2}\right\}-\left\{2 t_{2}\right\}\right) t_{1}+\left(2-2\left\{t_{1}\right\}-2\left\{t_{2}\right\}\right) t_{2}
$$

and

$$
\begin{aligned}
E_{[0]}\left(t_{1}, t_{2}\right)=1 & -\left\{t_{2}\right\}^{2}-\left\{2 t_{2}\right\}^{2}+2\left\{t_{1}\right\}\left\{\frac{t_{1}}{2}\right\}+2\left\{\frac{t_{1}}{2}+t_{2}\right\}\left\{t_{1}\right\} \\
& -\left\{\frac{t_{1}}{2}\right\}^{2}-2\left\{\frac{t_{1}}{2}+t_{2}\right\}^{2}-\left\{t_{1}\right\}-\left\{2 t_{2}\right\}-\left\{t_{1}\right\}^{2} \\
& +2\left\{2 t_{2}\right\}\left\{\frac{t_{1}}{2}+t_{2}\right\}+2\left\{t_{2}\right\}\left\{2 t_{2}\right\} .
\end{aligned}
$$

(3) Similarly, if $L$ is the vertical line, the sum of the lengths of vertical segments is given by the quasi-polynomial function

$$
S^{L}\left(t_{1}, t_{2}\right)=E_{[2]}\left(t_{1}, t_{2}\right)+E_{[1]}^{L}\left(t_{1}, t_{2}\right)+E_{[0]}^{L}\left(t_{1}, t_{2}\right)
$$

with

$$
\begin{aligned}
E_{[1]}^{L}\left(t_{1}, t_{2}\right)= & \left(\frac{1}{2}-\left\{\frac{t_{1}}{2}\right\}\right) t_{1}+\left(1-2\left\{t_{2}\right\}\right) t_{2} \\
E_{[0]}^{L}\left(t_{1}, t_{2}\right)=- & \left\{-\frac{t_{1}}{2}+t_{2}\right\}-\left\{\frac{t_{1}}{2}-t_{2}\right\}+\left\{t_{2}\right\}+\left\{\frac{t_{1}}{2}-t_{2}\right\}^{2} \\
& +\left\{\frac{t_{1}}{2}\right\}-\left\{\frac{t_{1}}{2}\right\}^{2}-\left\{t_{2}\right\}^{2}+\left\{-\frac{t_{1}}{2}+t_{2}\right\}^{2} .
\end{aligned}
$$

In contrast to the typical settings in the literature, we can allow the polytopes $\mathfrak{p}_{i}$ to be merely semi-rational, i.e., the facets of $\mathfrak{p}_{i}$ are parallel to rational hyperplanes, whereas the vertices are allowed to be arbitrary real points in $V$.

EXAMPLE 1.4. Let $\mathfrak{p}$ be the rectangle $0 \leqslant x \leqslant \sqrt{2}, 0 \leqslant y \leqslant 1$ (see Figure 3 ), a semi-rational polytope. ${ }^{(1)}$ For $t \in \mathbb{R}_{\geqslant 0}$, the number of lattice points in $t \mathfrak{p}$ is

$$
S(t \mathfrak{p}, 1)=(t-\{t\}+1)(\sqrt{2} t-\{\sqrt{2} t\}+1)=E_{2}(t) t^{2}+E_{1}(t) t+E_{0}(t)
$$

with coefficient functions

$$
\begin{aligned}
& E_{2}(t)=\sqrt{2}, \\
& E_{1}(t)=-\sqrt{2}\{t\}-\{\sqrt{2} t\}+\sqrt{2}+1, \\
& E_{0}(t)=(1-\{t\})(1-\{\sqrt{2} t\}) .
\end{aligned}
$$

Since the formulas of these functions involve both the rational step-linear function $\{t\}$ and the irrational step-linear function $\{\sqrt{2} t\}$, the coefficient functions are not periodic

\footnotetext{
${ }^{(1)}$ The reader is invited to follow the examples using our Maple programs, available at https: //www.math.ucdavis.edu/ latte/software/packages/maple/ and as part of LattE integrale, version 1.7 .2 and later.
} 
in $t$, but merely bounded functions of $t$. Functions of this type generalize quasipolynomials and are called semi-quasi-polynomials (the precise definition appears in Section 2.4). In the example, the function $S(t \mathfrak{p}, 1)$ is constant on the intersections ]$m, m+1[\cap] \frac{n}{\sqrt{2}}, \frac{n+1}{\sqrt{2}}[$ for $m, n$ positive integers.

More examples of (semi-)quasi-polynomial functions $S^{L}(t \mathfrak{p}, h)$ will be given later.

1.4. Second COntribution: Two families of APProximating multi-ParaMETER QUASI-POLYNOMIALS. We study the terms of highest polynomial degree of $S(\mathfrak{p}(b), h)$. We consider a patched weighted sum, i.e., a particular linear combination of intermediate weighted sums, for a finite family $\mathcal{L}_{k}^{\text {Barvinok }}$ of subspaces $L$ which was introduced by Barvinok in [7] (see Section 3.4 below for a definition). We thus obtain also a function of $b$ which is given by a quasi-polynomial on each chamber.

$$
S^{\mathcal{L}_{k}^{\text {Barvinok }}}(\mathfrak{p}(b), h)=\sum_{L \in \mathcal{L}_{k}^{\text {Barvinok }}} \rho(L) S^{L}(\mathfrak{p}(b), h)
$$

(where the constants $\rho(L)$ are defined in Section 3.1).

Furthermore, we introduce a new quasi-polynomial function of $b$, defined in a refined way by linear combinations of intermediate generating functions for the cones at vertices of $\mathfrak{p}(b)$. We denote it $S^{k, \text { cone-by-cone }}(\mathfrak{p}(b), h)$. This function is canonically defined due to the surprising analyticity of the cone-by-cone patched generating function (Proposition 4.2).

Our next main result is Theorem 5.3. We show that on each chamber the three quasi-polynomials, $b \mapsto S(\mathfrak{p}(b), h), b \mapsto S^{\mathcal{L}_{k}^{\text {Barvinok }}}(\mathfrak{p}(b), h)$ and $b \mapsto S^{k, \text { cone-by-cone }}$ $(\mathfrak{p}(b), h)$, have the same terms corresponding to the $k+1$ highest polynomial degrees. This result generalizes Barvinok [7] in several ways. Besides the introduction of the new $S^{k \text {,cone-by-cone }}(\mathfrak{p}(b), h)$, we allow any polynomial weight $h(x)$, while Barvinok considered only $h(x)=1$, and we write formulas in terms of quasi-polynomial functions of the real-valued multi-parameter $b$, while Barvinok considered a single polytope dilated by a positive integer.

$S^{k, \text { cone-by-cone }}(\mathfrak{p}(b), h)$ and $S^{\mathcal{L}_{k}^{\text {Barvinok }}}(\mathfrak{p}(b), h)$ involve subspaces $L$ of codimension $\leqslant k$. For such an $L$, the computation of $S^{L}(\mathfrak{p}(b), h)$ involves discrete sums over lattice points of semi-rational cones of dimension $\leqslant k$. For this reason, the quasi-polynomials $S^{\mathcal{L}_{k}^{\text {Barvinok }}}(\mathfrak{p}(b), h)$ and $S^{k, \text { cone-by-cone }}(\mathfrak{p}(b), h)$ are easier to compute than the original Ehrhart quasi-polynomial $S(\mathfrak{p}(b), h)$. Moreover, $S^{k, \text { cone-by-cone }}(\mathfrak{p}(b), h)$ is easier to compute than $S^{\mathcal{L}_{k}^{\text {Barvinok }}}(\mathfrak{p}(b), h)$.

Finally, when $\mathfrak{p}(b)$ is a simplex, we give an explicit formula for the coefficients $\rho(L)$ of the particular linear combination (5) of intermediate weighted sums used in Barvinok's approximation $S^{\mathcal{L}_{k}^{\text {Barvinok }}}(\mathfrak{p}(b), h)$ (Proposition 3.13), using an explicit formula for a Möbius function that was obtained by A. Björner and L. Lovász in a different context [11]

The precise statements of our main results will lead to explicit algorithms for computing both quasi-polynomials $S^{\mathcal{L}_{k}^{\text {Barvinok }}}(\mathfrak{p}(b), h)$ and $S^{k, \text { cone-by-cone }}(\mathfrak{p}(b), h)$ and thus the terms corresponding to the highest $k+1$ polynomial degrees of $S(\mathfrak{p}(b), h)$. In various interesting settings, for fixed $k$ and fixed chamber, as a corollary of our results, one can develop polynomial time algorithms to compute $S^{\mathcal{L}_{k}^{\text {Barvinok }}}(\mathfrak{p}(b), h)$ and $S^{k, \text { cone-by-cone }}(\mathfrak{p}(b), h)$; and thus two types of polynomial time algorithms to compute the terms corresponding to the highest expected $k+1$ polynomial degrees of $S(\mathfrak{p}(b), h)$ for a parametric simplex. Such polynomial time algorithms were the initial motivation for the study of these intermediate sums in our papers. However, in the present paper, 
in contrast to our previous papers $[2,3,5]$, we suppress detailed statements of such algorithms and their complexity.

We end our article by some explicit computations (obtained via a simple Maple program) of the quasi-polynomials $S^{k, \text { cone-by-cone }}(t \mathfrak{p}, 1)$ and $S^{\mathcal{L}_{k}^{\text {Barvinok }}}(t \mathfrak{p}, 1)$ for a dilated rational simplex $\mathfrak{p}$ in dimension $d \leqslant 4$, for $k \leqslant d$. For $k=0$ they both give the volume $t^{d} \operatorname{vol}(\mathfrak{p})$ and for $k=d$ they both give the number of lattice points of $t \mathfrak{p}$. For $1 \leqslant k \leqslant d-1, S^{\mathcal{L}_{k}^{\text {Barvinok }}}(t \mathfrak{p}, 1)$ and $S^{k, \text { cone-by-cone }}(t \mathfrak{p}, 1)$ have the same $k+1$ highest degree coefficients, but we see that they are actually different quasi-polynomials. It is not clear to us which one is the "best."

1.5. Techniques of this PAPER. Let us give the main ideas of our proofs.

We use the Brianchon-Gram decomposition of a polytope $\mathfrak{p}(b)$ as a signed sum of its supporting cones and the corresponding Brion formula, reducing the study of intermediate weighted sums $S^{L}(\mathfrak{p}(b), h)$ to that of intermediate generating functions $S^{L}(s+\mathfrak{c})(\xi)$ over tangent cones at vertices, which are defined in a similar way, by replacing $h(x)$ with an exponential function $x \mapsto \mathrm{e}^{\langle\xi, x\rangle}$. For $b$ in a chamber $\tau$, the vertices of $\mathfrak{p}(b)$ can be indexed, $s_{1}(b), \ldots, s_{r}(b)$, in such a way that for each index $j$, the cone of feasible directions at vertex $s_{j}(b)$ does not depend on $b$. Furthermore, the vertex $s_{j}(b)$ depends linearly on the parameter $b$. This fact is the basis of all the constructions and results of the present paper. We use our previous results on semi-rational affine polyhedral cones in [4] and [5], where we studied in detail the intermediate generating functions of a shifted cone $S^{L}(s+\mathfrak{c})(\xi)$ (where $\mathfrak{c}$ is a fixed polyhedral rational cone) as a function of $s$ and $\xi$. Theorem 5.3 uses the results of [4] on the bidegree structure, i.e., the interplay between the local degree with respect to $s$ and the homogeneous degree with respect to $\xi$. Also, as in Barvinok's Fourier inversion method in [7], the Poisson summation formula for $S^{L}(s+\mathfrak{c})(\xi)$ obtained in [4] is crucial to the proof of Theorem 5.3.

\section{INTERMEDIATE WEIGHTED EHRHART QUASI-POLYNOMIALS FOR PARAMETRIC POLYTOPES}

2.1. Notations. In this paper, $V$ is a vector space over $\mathbb{R}$ of dimension $d$. The running element of $V$ is denoted by $x$. As usual, the dual vector space is denoted by $V^{*}$. By $V_{\mathbb{C}}^{*}$ we denote the complexified dual space. The running element of $V^{*}$ or $V_{\mathbb{C}}^{*}$ is denoted by $\xi$.

The vector space $V$ is endowed with a lattice $\Lambda$ that spans $V$ (one says that $V$ is rational). We denote by $\Lambda^{*}$ the dual lattice in $V^{*}$. We denote by $V_{\mathbb{Q}}=\Lambda \otimes \mathbb{Q}$ and $V_{\mathbb{Q}}^{*}=\Lambda^{*} \otimes \mathbb{Q}$ the sets of rational elements of $V$ and $V^{*}$, respectively. A subspace $L$ of $V$ is called rational if $L \cap \Lambda$ is a lattice in $L$. If $L$ is a rational subspace, the image of $\Lambda$ in $V / L$ is a lattice in $V / L$, so that $V / L$ is a rational vector space. The image of $\Lambda$ in $V / L$ is called the projected lattice. It is denoted by $\Lambda_{V / L}$. A rational space $V$, with lattice $\Lambda$, has a canonical Lebesgue measure $\mathrm{d} x=\mathrm{d} m_{\Lambda}(x)$, for which $V / \Lambda$ has measure 1. We denote by $L^{\perp} \subset V^{*}$ the space of linear forms $\xi \in V^{*}$ which vanish on $L$.

An affine subspace of $V$ is called semi-rational if it can be written as $s+L$ where $L$ is a rational subspace and $s$ is any point of $V$.

The polyhedra of this study are subsets of $V$. A polyhedron is the intersection of a finite number of closed halfspaces. A polytope is a compact polyhedron, not necessarily full-dimensional.

The faces of a polyhedron $\mathfrak{p}$ can be of dimension 0 (vertices), 1 (edges),..., $\operatorname{dim}(\mathfrak{p})-1$ (facets), and $\operatorname{dim}(\mathfrak{p})$ (the polyhedron $\mathfrak{p}$ itself). A wall of a polyhedron $\mathfrak{p}$ is a hyperplane $H$ such that $\mathfrak{p}$ is on one side of $H$ and $\operatorname{dim}(H \cap \mathfrak{p})=\operatorname{dim}(\mathfrak{p})-1$. 
Then $H \cap \mathfrak{p}$ is called a facet of $\mathfrak{p}$. A polytope $\mathfrak{p}$ of dimension $d$ is called simple if each vertex $s$ belongs to exactly $d$ facets.

If $\mathfrak{f} \subset V$ is a polyhedron, the subspace $\operatorname{lin}(\mathfrak{f})$ is defined as the linear subspace of $V$ generated by $p-q$ for $p, q \in \mathfrak{f}$. A polyhedron $\mathfrak{p}$ is called semi-rational if $\operatorname{lin}(\mathfrak{f})$ is rational for all facets $\mathfrak{f}$ of $\mathfrak{p}$.

In this article, a cone is a convex polyhedral rational cone (with vertex 0) and an affine cone is the shifted set $s+\mathfrak{c}$ of a rational cone $\mathfrak{c}$ for some $s \in V$. A cone $\mathfrak{c}$ is called pointed if it does not contain a line. A cone $\mathfrak{c}$ is called simplicial if it is generated by linearly independent elements of $V$. A simplicial cone $\mathfrak{c}$ is called unimodular if it is generated by independent lattice vectors $v_{1}, \ldots, v_{k}$ such that $\left\{v_{1}, \ldots, v_{k}\right\}$ is part of a basis of $\Lambda$. An affine cone $s+\mathfrak{c}$ is called pointed (simplicial, unimodular, respectively) if the associated cone $\mathfrak{c}$ is.

The set of vertices of a polytope $\mathfrak{p}$ is denoted by $\mathcal{V}(\mathfrak{p})$. For each vertex $s$, the cone of feasible directions at $s$ is denoted by $\mathfrak{c}_{s}$.

When we speak of the parametric polytope $\mathfrak{p}(b)$, the parameter space is $\mathbb{R}^{N}$. Its running element is denoted by $b=\left(b_{1}, b_{2}, \ldots, b_{N}\right)$. We denote by $e_{j}$ the canonical basis of $\mathbb{R}^{N}$, and write also $b=\sum_{j=1}^{N} b_{j} e_{j}$.

The indicator function of a subset $E$ is denoted by $[E]$.

For $t \in \mathbb{R}$, we denote by $\{t\} \in[0,1[$ the fractional part of $t$. Then $t-\{t\}$ is an integer.

2.2. INTERMEDIATE WEIGHTED SUMS AND GENERATING FUNCTIONS ON POLYHEDRA. Let $\mathfrak{p} \subset V$ be a semi-rational polytope, $L$ a rational subspace of $V$ and let $h$ be a polynomial function on $V$. We are interested in the properties of the intermediate weighted sum on $\mathfrak{p}$,

$$
S^{L}(\mathfrak{p}, h)=\sum_{y \in \Lambda_{V / L}} \int_{\mathfrak{p} \cap(y+L)} h(x) \mathrm{d} x .
$$

Here, $\Lambda_{V / L}$ is the projected lattice and $\mathrm{d} x$ is the Lebesgue measure on $y+L$ defined by the intersection lattice $L \cap \Lambda$. Thus we integrate over the intersections of $\mathfrak{p}$ with subspaces parallel to a fixed rational subspace $L$ through all lattice points, and sum the integrals.

Although $S^{L}(\mathfrak{p}, h)$ depends on the lattice $\Lambda$, we do not indicate this dependence in the notation.

$S^{L}(\mathfrak{p}, h)$ interpolates between the integral

$$
I(\mathfrak{p}, h)=\int_{\mathfrak{p}} h(x) \mathrm{d} x
$$

of $h$ on $\mathfrak{p}$, which corresponds to $L=V$, and the discrete weighted sum

$$
S(\mathfrak{p}, h)=\sum_{x \in \mathfrak{p} \cap \Lambda} h(x),
$$

which corresponds to $L=\{0\}$.

Consider, instead of the polynomial function $h(x)$, the exponential function $\mathrm{e}^{\langle\xi, x\rangle}$. Thus we define the following holomorphic function of $\xi$ :

$$
S^{L}(\mathfrak{p})(\xi)=\sum_{y \in \Lambda_{V / L}} \int_{\mathfrak{p} \cap(y+L)} \mathrm{e}^{\langle\xi, x\rangle} \mathrm{d} x .
$$

Following the method initiated by Barvinok, the study of the generating function $S^{L}(\mathfrak{p})(\xi)$ reduces to the computation of the similar functions $S^{L}(\mathfrak{u})(\xi)$, where $\mathfrak{u}$ are affine cones, see $[2,3,6]$, etc. However, for an arbitrary non-compact polyhedron $\mathfrak{p}$, the above definition $(7)$ of $S^{L}(\mathfrak{p})(\xi)$ makes sense only as a generalized function of $i \xi$. 
To avoid the use of distributions, and stay in an algebraic context, we will define $S^{L}(\mathfrak{p})(\xi)$, for any semi-rational polyhedron $\mathfrak{p}$ as a meromorphic function of $\xi$, satisfying a valuation property.

Let us recall the notations of [4, Definitions 2.1-2.3].

DEFINITION 2.1.

(1) We denote by $\mathcal{M}_{\ell}\left(V^{*}\right)$ the ring of meromorphic functions around $0 \in V_{\mathbb{C}}^{*}$ which can be written as a quotient $\frac{\phi(\xi)}{\prod_{j=1}^{N}\left\langle\xi, w_{j}\right\rangle}$, where $\phi(\xi)$ is holomorphic near 0 and $w_{j}$ are non-zero elements of $V$ in finite number. (The subscript $\ell$ is mnemonic for the linear forms that appear in the denominator.)

(2) We denote by $\mathcal{R}_{[\geqslant m]}\left(V^{*}\right)$ the space of rational functions which can be written as $\frac{P(\xi)}{\prod_{j=1}^{N}\left\langle\xi, w_{j}\right\rangle}$, where $P$ is a homogeneous polynomial of degree greater or equal to $m+N$. These rational functions are said to be homogeneous of degree at least $m$.

(3) We denote by $\mathcal{R}_{[m]}\left(V^{*}\right)$ the space of rational functions which can be written as $\frac{P(\xi)}{\prod_{j=1}^{N}\left\langle\xi, w_{j}\right\rangle}$, where $P$ is homogeneous of degree $m+N$. These rational functions are said to be homogeneous of degree $m$.

Definition 2.2. For $\phi \in \mathcal{M}_{\ell}\left(V^{*}\right)$, the homogeneous component $\phi_{[m]}$ of degree $m$ of $\phi$ is defined by considering $\phi(\tau \xi)$ as a meromorphic function of one variable $\tau \in \mathbb{C}$, with Laurent series expansion

$$
\phi(\tau \xi)=\sum_{m \geqslant m_{0}} \tau^{m} \phi_{[m]}(\xi) .
$$

Thus $\phi_{[m]} \in \mathcal{R}_{[m]}\left(V^{*}\right)$.

Definition 2.3. An $\mathcal{M}_{\ell}\left(V^{*}\right)$-valued valuation on the set of semi-rational polyhedra $\mathfrak{p} \subseteq V$ is a map $F$ from this set to the vector space $\mathcal{M}_{\ell}\left(V^{*}\right)$ such that whenever the indicator functions $\left[\mathfrak{p}_{i}\right]$ of a family of polyhedra $\mathfrak{p}_{i}$ satisfy a linear relation $\sum_{i} r_{i}\left[\mathfrak{p}_{i}\right]=0$, then the elements $F\left(\mathfrak{p}_{i}\right)$ satisfy the same relation

$$
\sum_{i} r_{i} F\left(\mathfrak{p}_{i}\right)=0
$$

We recall the following result from [3, Proposition 19] (see also [5, Proposition 3] and [4, Proposition 2.4]).

Proposition 2.4. Let $L \subseteq V$ be a rational subspace. There exists a unique valuation which associates a meromorphic function $S^{L}(\mathfrak{p})(\xi)$ belonging to $\mathcal{M}_{\ell}\left(V^{*}\right)$ to every semi-rational polyhedron $\mathfrak{p} \subseteq V$, so that the following properties hold:

(1) If $\mathfrak{p}$ contains a line, then $S^{L}(\mathfrak{p})=0$.

(2) $S^{L}(\mathfrak{p})(\xi)=\sum_{y \in \Lambda_{V / L}} \int_{\mathfrak{p} \cap(y+L)} \mathrm{e}^{\langle\xi, x\rangle} \mathrm{d} x$, for every $\xi \in V^{*}$ such that this sum converges.

We remark that property (1) above reflects the fact that the distribution $t \mapsto$ $\sum_{n \in \mathbb{Z}} \mathrm{e}^{i n t}$ is supported on $2 \pi \mathbb{Z}$.

Following [3, Section 3.4], [5, Section 2.2] and [4, Definition 2.5], the function $S^{L}(\mathfrak{p})(\xi)$ will be called the intermediate generating function of the polyhedron $\mathfrak{p}$.

If $\mathfrak{p}$ is a polytope, then clearly the homogeneous component of degree $r$ of $S^{L}(\mathfrak{p})(\xi)$ is given by

$$
S^{L}(\mathfrak{p})_{[r]}(\xi)=\sum_{y \in \Lambda_{V / L}} \int_{\mathfrak{p} \cap(y+L)} \frac{\langle\xi, x\rangle^{r}}{r !} \mathrm{d} x .
$$


Thus the intermediate generating function $S^{L}(\mathfrak{p})(\xi)$ of the polytope $\mathfrak{p}$ allows us to compute $S^{L}(\mathfrak{p}, h)$ for any polynomial $h$ by decomposing $h$ in sums of powers of linear forms [2, Section 3.4].

The intermediate generating function $S^{L}(\mathfrak{p})(\xi)$ interpolates between the integral (continuous generating function)

$$
I(\mathfrak{p})(\xi)=\int_{\mathfrak{p}} \mathrm{e}^{\langle\xi, x\rangle} \mathrm{d} x
$$

which corresponds to $L=V$, and the discrete sum (discrete generating function)

$$
S(\mathfrak{p})(\xi)=\sum_{x \in \mathfrak{p} \cap \Lambda} \mathrm{e}^{\langle\xi, x\rangle}
$$

which corresponds to $L=\{0\}$.

We introduced in [4, Definition 2.18] the algebra $\mathcal{Q} \mathcal{P}^{\Psi}(V)$ of quasi-polynomial functions on $V$ defined as follows. (These definitions are analogues of the quasi-polynomial functions on $\mathbb{R}^{N}$ defined in the introduction.)

Definition 2.5. Let $\Psi$ be a finite subset of elements of $V_{\mathbb{Q}}^{*}$. Define the algebra $\mathcal{Q}^{\Psi}(V)$ of functions of $s \in V$ generated by the functions $s \mapsto\{\langle\gamma, s\rangle\}$ with $\gamma \in \Psi$. A function $f$ will be called a (rational) step-polynomial function on $V$, if $f$ belongs to $\mathcal{Q}^{\Psi}(V)$ for some $\Psi$.

Such a function $f$ is bounded and periodic modulo $q \Lambda$ for some integer $q$.

The algebra $\mathcal{Q}^{\Psi}(V)$ again has a natural filtration, where $\mathcal{Q}_{[\leqslant k]}^{\Psi}(V)$ is the subspace spanned by products of at most $k$ functions $\{\langle\gamma, s\rangle\}$. The algebra $\mathcal{Q} \mathcal{P}^{\Psi}(V)$ is the algebra generated by $\mathcal{Q}^{\Psi}(V)$ and ordinary polynomial functions. A function $f$ will be called a quasi-polynomial function on $V$, if $f$ belongs to $\mathcal{Q P} \mathcal{P}^{\Psi}(V)$ for some $\Psi$.

Let $\mathfrak{c}$ be a fixed cone. The translated cone $s+\mathfrak{c}$ is a semi-rational polyhedron. Let $S^{L}(s+\mathfrak{c})_{[m]}(\xi)$ be the degree- $m$ homogeneous component of the functions $S^{L}(s+\mathfrak{c})(\xi)$. The lowest homogeneous component of $S^{L}(s+\mathfrak{c})$ has degree $-d$ and is equal to $I(\mathfrak{c})(\xi)$, as proved in [4, Theorem 2.25 (iii)].

Remark that for any $v \in \Lambda+L$,

$$
S^{L}(v+s+\mathfrak{c})(\xi)=\mathrm{e}^{\langle\xi, v\rangle} S^{L}(s+\mathfrak{c})(\xi) .
$$

Thus the function

$$
M^{L}(s, \mathfrak{c})(\xi)=\mathrm{e}^{-\langle\xi, s\rangle} S^{L}(s+\mathfrak{c})(\xi)
$$

is a function of $s \in V /(\Lambda+L)$.

Let $M^{L}(s, \mathfrak{c})_{[m]}$ be the degree- $m$ homogeneous component of the function $M^{L}(s, \mathfrak{c})$. The function $s \mapsto M^{L}(s, \mathfrak{c})_{[m]}$ is a periodic function of the variable $s \in V$ with values in the space $\mathcal{R}_{[m]}\left(V^{*}\right)$. Results of [4] on the nature of this function will be used in Section 2.4.

Note the obvious, but fundamental formula:

$$
S^{L}(s+\mathfrak{c})_{[m]}(\xi)=\sum_{r=0}^{m+d} M^{L}(s, \mathfrak{c})_{[m-r]}(\xi) \frac{\langle\xi, s\rangle^{r}}{r !}
$$

REMARK 2.6. Equation (9) expresses the function $s \mapsto S^{L}(s+\mathfrak{c})_{[m]}(\xi)$ as a quasipolynomial function of the variable $s \in V$ with values in the space $\mathcal{R}_{[m]}\left(V^{*}\right)$. Indeed $s \mapsto M^{L}(s, \mathfrak{c})_{[m-r]}(\xi)$ is a periodic function on $V$ given by a step-polynomial formula, while $s \mapsto \frac{\langle\xi, s\rangle^{r}}{r !}$ is a polynomial function of $s$. 

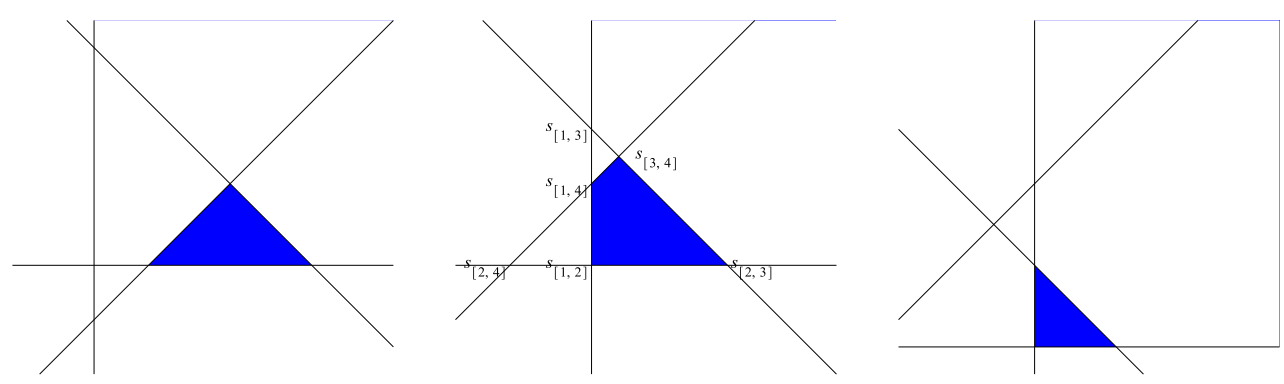

Figure 5. Example 2.8, $\mathfrak{p}(b)$ for $b=(2,0,0,6),(0,0,5,3),(0,3,3,0)$.

The lowest degree homogeneous component of $M^{L}(s, \mathfrak{c})$ is the same as that of $S^{L}(s+\mathfrak{c})$, hence of degree $-d$ and equal to $I(\mathfrak{c})(\xi)$.

$$
M^{L}(s, \mathfrak{c})_{[-d]}=I(\mathfrak{c})(\xi) .
$$

When $L=\{0\}$, we write $S(s+\mathfrak{c})$ instead of $S^{\{0\}}(s+\mathfrak{c})$ and $M(s, \mathfrak{c})$ instead of $M^{\{0\}}(s, \mathfrak{c})$.

\subsection{Parametric polytopes.}

2.3.1. Chambers. First, we recall some well known notions about parametric polytopes (cf. for instance [18]). Let $\alpha=\left(\alpha_{1}, \alpha_{2}, \ldots, \alpha_{N}\right)$ be a list of $N$ elements of $\Lambda^{*}$ such that the cone generated by $\alpha$ is the whole space $V^{*}$. For $b=\left(b_{1}, b_{2}, \ldots, b_{N}\right) \in \mathbb{R}^{N}$, let

$$
\mathfrak{p}(\alpha, b)=\left\{x \in V:\left\langle\alpha_{j}, x\right\rangle \leqslant b_{j}, j=1, \ldots, N\right\} .
$$

Then $\mathfrak{p}(\alpha, b)$ is a semi-rational polytope. We will often denote it simply by $\mathfrak{p}(b)$.

It is clear that if we dilate the parameter $b$ in $t b$ with $t \geqslant 0$, we obtain the dilated polytope $t \mathfrak{p}(b)$.

$$
t \mathfrak{p}(b)=\mathfrak{p}(t b) .
$$

We denote also by $\alpha$ the map $V \rightarrow \mathbb{R}^{N}$ given by

$$
\alpha(x)=\left(\left\langle\alpha_{1}, x\right\rangle,\left\langle\alpha_{2}, x\right\rangle, \ldots,\left\langle\alpha_{N}, x\right\rangle\right) .
$$

If $v_{0} \in V$, the shifted polytope $\mathfrak{p}(b)+v_{0}$ is given by

$$
\mathfrak{p}(b)+v_{0}=\mathfrak{p}\left(b+\alpha\left(v_{0}\right)\right) .
$$

Hence, for many aspects of parametric polytopes, the relevant space of parameters is not $\mathbb{R}^{N}$ itself, but the quotient space $\mathbb{R}^{N} / \alpha(V)$ (see Remark 2.32). For instance, $\mathfrak{p}(b)$ is not empty if and only if $b$ lies in the closed cone generated by the standard basis $e_{i}$ of $\mathbb{R}^{N}$ and the subspace $\alpha(V)$. Indeed, for $x \in \mathfrak{p}(b), b=\sum_{j}\left(b_{j}-\left\langle\alpha_{j}, x\right\rangle\right) e_{j}+\alpha(x)$.

EXAmPle 2.7. The simplest example is $V=\mathbb{R}$ with $\alpha_{1}=x, \alpha_{2}=-x$. Thus $\mathfrak{p}(b)=$ $\left\{x \in \mathbb{R}, x \leqslant b_{1},-x \leqslant b_{2}\right\}$. If $b_{1}+b_{2}<0, \mathfrak{p}(b)$ is empty, otherwise it is the interval $\left[-b_{2}, b_{1}\right]$.

ExAmPLe 2.8 (Figure 5). Let $V=\mathbb{R}^{2}$ and $\alpha=\left(-x_{1},-x_{2}, x_{1}+x_{2},-x_{1}+x_{2}\right)$. Then $\mathfrak{p}(b)$ is defined by the inequalities

$$
-x_{1} \leqslant b_{1}, \quad-x_{2} \leqslant b_{2}, \quad x_{1}+x_{2} \leqslant b_{3}, \quad-x_{1}+x_{2} \leqslant b_{4} .
$$

Figure 5 displays this polygon for three values of $b$, one for each of the three combinatorial types which occur in this example. (See below Example 2.15.) 
When $b$ varies, the combinatorial type of $\mathfrak{p}(b)$ changes. The $\alpha$-chambers $\tau \subset \mathbb{R}^{N}$, defined below, are conical open subsets such that the combinatorial type of $\mathfrak{p}(b)$ does not change when $b$ runs over $\tau$. First, we want to index the vertices of $\mathfrak{p}(b)$. For this purpose, we introduce the following subsets of $\{1, \ldots, N\}$.

Definition 2.9. $\mathcal{B}$ is the set of subsets $B \subseteq\{1, \ldots, N\}$ such that $\left(\alpha_{j}, j \in B\right)$ is a basis of $V^{*}$.

For $B \in \mathcal{B}$, the linear map $b \mapsto s_{B}(b): \mathbb{R}^{N} \rightarrow V$ is defined by

$$
\left\langle\alpha_{j}, s_{B}(b)\right\rangle=b_{j} \text { for } j \in B .
$$

For $B \in \mathcal{B}$, the cone $\mathfrak{c}_{B} \subset V$ is defined by

$$
\mathfrak{c}_{B}=\left\{x \in V:\left\langle\alpha_{j}, x\right\rangle \leqslant 0, \text { for } j \in B\right\} .
$$

Thus, the point $s_{B}(b)$ is the intersection of the $d$ linearly independent hyperplanes $\left\langle\alpha_{j}, s_{B}(b)\right\rangle=b_{j}$ for $j \in B$. The points $s_{B}(b), B \in \mathcal{B}$, are classically called the vertices of the arrangement of hyperplanes $\left\langle\alpha_{j}, x\right\rangle=b_{j}$.

ExAmple 2.10 (Continuation of Example 2.8, Figure 5). Here, $\mathcal{B}$ consists of all pairs $[i, j]$ with $1 \leqslant i<j \leqslant 4$. The six points $s_{B}(b)$ are the intersections of the corresponding two lines:

$$
\begin{aligned}
s_{[1,2]}(b) & =\left(-b_{1},-b_{2}\right), & s_{[1,3]}(b) & =\left(-b_{1}, b_{1}+b_{3}\right), \\
s_{[1,4]}(b) & =\left(-b_{1},-b_{1}+b_{4}\right), & s_{[2,3]}(b) & =\left(b_{2}+b_{3},-b_{2}\right), \\
s_{[2,4]}(b) & =\left(-b_{2}-b_{4},-b_{2}\right), & s_{[3,4]}(b) & =\left(\frac{1}{2}\left(b_{3}-b_{4}\right), \frac{1}{2}\left(b_{3}+b_{4}\right)\right) .
\end{aligned}
$$

The six cones $\mathfrak{c}_{B}$ (up to a shift) appear as the tangent cones of the vertices of the three example polytopes $\mathfrak{p}(b)$ in Figure 5 .

The actual vertices of the polytope $\mathfrak{p}(b)$ are exactly those points $s_{B}(b)$ which satisfy the remaining inequalities $\left\langle\alpha_{k}, s_{B}(b)\right\rangle \leqslant b_{k}$ for $k \notin B$. Thus, for $B \in \mathcal{B}$, we consider the following cone in $\mathbb{R}^{N}$.

$$
\mathfrak{d}_{B}=\left\{b \in \mathbb{R}^{N}: b_{k}-\left\langle\alpha_{k}, s_{B}(b)\right\rangle \geqslant 0, \text { for } k \notin B\right\} .
$$

Lemma 2.11. Let $e_{j}, 1 \leqslant j \leqslant N$ be the standard basis of $\mathbb{R}^{N}$, and let $\phi_{j}$ be the projection of $e_{j}$ on $\mathbb{R}^{N} / \alpha(V)$ for $1 \leqslant j \leqslant N$.

(1) The cone $\mathfrak{d}_{B}$ coincides with $\alpha(V)+\sum_{k \notin B} \mathbb{R}_{\geqslant 0} e_{k}$.

(2) The projection of $\mathfrak{d}_{B}$ on $\mathbb{R}^{N} / \alpha(V)$ is the simplicial cone with generators $\phi_{k}$, $k \notin B$.

Proof. Part (1) follows from the formula

$$
b-\alpha\left(s_{B}(b)\right)=\sum_{k \notin B}\left(b_{k}-\left\langle\alpha_{k}, s_{B}(b)\right\rangle\right) e_{k} .
$$

For (2), note that if $B$ is a subset of $d$ elements of $\{1, \ldots, N\}$, the linear forms $\left(\alpha_{j}, j \in B\right)$ are linearly independent if and only if the vectors $\left(\phi_{k}, k \notin B\right)$ are.

DEFINITION 2.12. An $\alpha$-chamber $\tau \subset \mathbb{R}^{N}$ is a connected component of the complement of the union of the boundaries of the cones $\mathfrak{d}_{B}$ (13) for all $B \in \mathcal{B}$.

Thus, for every $j \in\{1, \ldots, N\}$ and $B \in \mathcal{B}$, the linear form $b_{j}-\left\langle\alpha_{j}, s_{B}(b)\right\rangle$ keeps a constant sign on every chamber.

There is a unique chamber $\tau_{e}$ such that $\mathfrak{p}(b)$ is empty for $b \in \tau_{e}$, it is the complement of the cone $\alpha(V)+\sum_{k=1}^{N} \mathbb{R}_{\geqslant 0} e_{k}$. The other chambers are contained in $\alpha(V)+\sum_{k=1}^{N} \mathbb{R}_{\geqslant 0} e_{k}$, they are called admissible. 


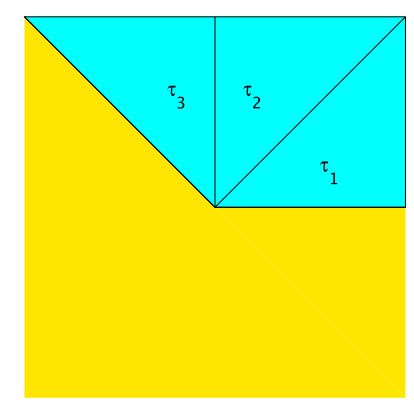

FiguRE 6. Four projected chambers in Example 2.15.

Chambers are open conical sets which contain the vector subspace $\alpha(V)$. Thus the relevant sets are the projected chambers in $\mathbb{R}^{N} / \alpha(V)$. They are easier to visualize.

LEMMA 2.13. The projected chambers in $\mathbb{R}^{N} / \alpha(V)$ are the connected components of the complement of the union of the boundaries of the cones generated by $N-d$ linearly independent vectors among the $\phi_{j}$ 's.

We have the following well-known description of the walls, vertices and cones at vertices of $\mathfrak{p}(b)$ when $b$ varies in an $\alpha$-chamber.

Proposition 2.14. Let $\tau$ be an $\alpha$-chamber. Let $\mathcal{B}_{\tau}$ be the set of $B \in \mathcal{B}$ such that $\tau$ is contained in the cone $\mathfrak{d}_{B}$. Then, for $b \in \tau$, the following holds.

(1) $\mathfrak{p}(b)$ is simple.

(2) The hyperplane $\left\langle\alpha_{j}, x\right\rangle=b_{j}$ is a wall of $\mathfrak{p}(b)$, i.e., its intersection with $\mathfrak{p}(b)$ is a facet, if and only if $j$ belongs to some $B$ in $\mathcal{B}_{\tau}$.

(3) The vertices of $\mathfrak{p}(b)$ are the points $s_{B}(b)$ where $B$ runs over $\mathcal{B}_{\tau}$.

(4) For $B \in \mathcal{B}_{\tau}$, the cone of feasible directions at vertex $s_{B}(b)$ of $\mathfrak{p}(b)$ is the cone $\mathfrak{c}_{B}=\left\{x \in V:\left\langle\alpha_{j}, x\right\rangle \leqslant 0\right.$ for $\left.j \in B\right\}$. In particular, it depends only on $B$, not on $b$.

The projection $\tau / \alpha(V)$ of the chamber $\tau$ on $\mathbb{R}^{N} / \alpha(V)$ is the intersection of all the simplicial cones, generated by subsets of the $\phi_{k}$, and containing it. Thus an equation $\alpha_{j}$ is redundant for $\mathfrak{p}(b)$ if and only if the vector $\phi_{j}$ is an edge of all the simplicial cones $\mathfrak{d}_{B} / \alpha(V)$, containing $\tau / \alpha(V)$.

ExAmple 2.15 (Continuation of Example 2.8 and Figure 5 ). Here, $\Phi=\left(\phi_{1}, \phi_{2}, \phi_{3}=\right.$ $\left.\frac{\phi_{1}+\phi_{2}}{2}, \phi_{4}=\frac{-\phi_{1}+\phi_{2}}{2}\right)$. There are four chambers, whose projections are represented in Figure 6 . The projections of the three admissible chambers $\tau_{i}, i=1,2,3$ are colored in blue. The fourth chamber (for which $\mathfrak{p}(b)$ is empty) is colored in yellow. $\tau_{1}$ is the cone $\left\{b: \sum_{j=1}^{4} b_{j} \phi_{j} \in \mathbb{R}_{>0} \phi_{1}+\mathbb{R}_{>0} \phi_{3}\right\} . \tau_{2}$ is the cone $\left\{b: \sum_{j=1}^{4} b_{j} \phi_{j} \in \mathbb{R}_{>0} \phi_{2}+\mathbb{R}_{>0} \phi_{3}\right\}$. $\tau_{3}$ is the cone $\left\{b: \sum_{j=1}^{4} b_{j} \phi_{j} \in \mathbb{R}_{>0} \phi_{2}+\mathbb{R}_{>0} \phi_{4}\right\}$.

For instance, $\tau_{2}$ is defined by the inequalities $-b_{1}+b_{2}+b_{4}>0,2 b_{1}+b_{3}-b_{4}>0$.

As we see on Figure 5 , for $b \in \tau_{1}, \mathfrak{p}(b)$ is a triangle, for $b \in \tau_{2}$, it is a quadrilateral, for $b \in \tau_{3}$, it is again a triangle.

We have $\mathcal{B}_{\tau_{1}}=\{[2,3],[2,4],[3,4]\}, \mathcal{B}_{\tau_{2}}=\{[1,2],[1,4],[2,3],[3,4]\}$, and $\mathcal{B}_{\tau_{3}}=$ $\{[1,2],[1,3],[2,3]\}$.

The index 1 does not belong to the union of the sets $B$ when $B$ varies in $\mathcal{B}_{\tau_{1}}$, thus the condition $-x_{1} \leqslant b_{1}$ is redundant for the polytope $\mathfrak{p}(b)$ when $b \in \tau_{1}$, as seen on Figure 5.

Similarly, the condition $-x_{1}+x_{2} \leqslant b_{4}$ is redundant for the polytope $\mathfrak{p}(b)$ when $b \in \tau_{3}$. 


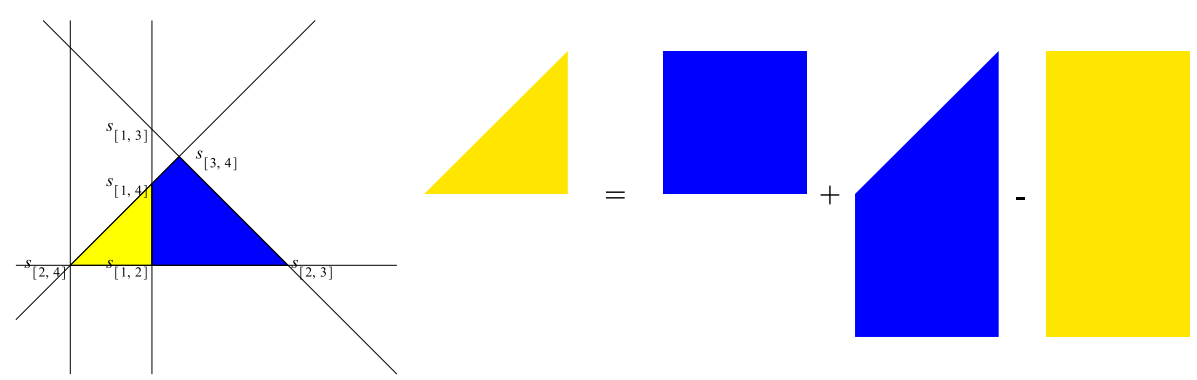

Figure 7. Left, in the parametric polytope of Examples 2.8 and 2.15, as the face $-x_{1}=b_{1}$ moves to the left, for $b \in \bar{\tau}_{2}$, the vertex $s_{[2,4]}$ merges with vertices $s_{[1,2]}$ and $s_{[1,4]}$, when $b$ reaches the boundary of the chamber $\tau_{2}$ and the quadrilateral degenerates to a triangle. Right, the indicator function of the supporting cone of $\mathfrak{p}(b)$ at vertex $s_{[2,4]}(b)=s_{[1,2]}(b)=s_{[1,4]}($ yellow $)$ is the sum of the indicator functions of the cones $s_{[1,2]}(b)+\mathfrak{c}_{[1,2]}$ and $s_{[1,4]}(b)+\mathfrak{c}_{[1,4]}($ blue $)$, modulo the indicator function of an affine cone with a line (yellow).

For $b \in \tau_{2}$, the four equations $\left\langle\alpha_{j}, x\right\rangle=b_{j}$ define facets of the quadrilateral $\mathfrak{p}(b)$.

If $b$ lies in the boundary of an admissible chamber $\tau$, then all vertices of $\mathfrak{p}(b)$ are of the form $s_{B}(b)$, for $B \in \mathcal{B}_{\tau}$, but several $B \in \mathcal{B}_{\tau}$ may give the same vertex $s_{B}(b)$.

REMARK 2.16 (Minkowski sums of polytopes). Remark finally the following relation between parametric polytopes and Minkowski sums $t_{1} \mathfrak{p}_{1}+t_{2} \mathfrak{p}_{2}+\cdots+t_{q} \mathfrak{p}_{q}$ of polytopes. Let $\tau$ be an admissible chamber. Let $b_{1}, b_{2}, \ldots, b_{q}$ in $\bar{\tau}$ and $t_{1} \geqslant 0, \ldots, t_{q} \geqslant 0$, then $t_{1} b_{1}+t_{2} b_{2}+\cdots+t_{q} b_{q}$ is in $\bar{\tau}$, and

$$
t_{1} \mathfrak{p}\left(b_{1}\right)+t_{2} \mathfrak{p}\left(b_{2}\right)+\cdots+t_{q} \mathfrak{p}\left(b_{q}\right)=\mathfrak{p}\left(t_{1} b_{1}+\cdots+t_{q} b_{q}\right) .
$$

Indeed, using the linearity of the map $b \mapsto s_{B}(b)$, we see immediately that any point in the convex hull of the elements $s_{B}\left(t_{1} b_{1}+\cdots+t_{q} b_{q}\right)$ with $B \in \mathcal{B}_{\tau}$ is a sum of points in $\mathfrak{p}\left(t_{1} b_{1}\right), \ldots, \mathfrak{p}\left(t_{q} b_{q}\right)$.

Conversely (see Section 2.5.4), it can be shown that any Minkowski linear sum $t_{1} \mathfrak{p}_{1}+t_{2} \mathfrak{p}_{2}+\cdots+t_{q} \mathfrak{p}_{q}$ can be embedded in a parametric family of polytopes.

REMARK 2.17 (Wall crossing). One of the interest of parametric polytopes is that we can also observe the variation of $\mathfrak{p}(b)$ (see Figure 5), when the parameter $b$ crosses a wall of a chamber $\tau$. This corresponds to flips of the corresponding toric varieties. The variation of the set $\mathfrak{p}(b)$ has been studied in detail in [10]. In this article we will only be concerned with the behavior of the function $b \mapsto S^{L}(\mathfrak{p}(b), h)$ when $b$ runs in the closure of a fixed admissible chamber.

2.3.2. Brion's theorem on supporting cones at vertices. The basis of the present article is the following theorem, which follows from the Brianchon-Gram decomposition of a polytope $\mathfrak{p}(b)$.

Proposition 2.18. The indicator function of a polytope is equal to the sum of the indicator functions of its supporting cones at vertices, modulo linear combinations of indicator functions of affine cones with lines.

For parametric polytopes, combined with the above description of cones at vertices, this gives a decomposition of the indicator function $[\mathfrak{p}(b)]$ for $b \in \tau$. If $b$ lies in the boundary of an admissible chamber $\tau$, then several $B \in \mathcal{B}_{\tau}$ may give the same 
vertex $s_{B}(b)$. Nevertheless, when $b$ lies in the closure $\bar{\tau}$, then modulo indicator functions of affine cones with lines, the indicator function of the supporting cone of $\mathfrak{p}(b)$ at vertex $s_{B}(b)$ is the sum of the indicator functions of the cones $s_{B}(b)+\mathfrak{c}_{B}$ for all the $B \in \mathcal{B}_{\tau}$ which give this vertex (Figure 7 ). One way to prove it is to use the continuity of the Brianchon-Gram decomposition of [p(b)], proven in [10].

Proposition 2.19. Let $\tau$ be an admissible $\alpha$-chamber with closure $\bar{\tau}$. For $b \in \bar{\tau}$, the indicator function of $\mathfrak{p}(b)$ is given by

$$
[\mathfrak{p}(b)] \equiv \sum_{B \in \mathcal{B}_{\tau}}\left[s_{B}(b)+\mathfrak{c}_{B}\right] \text { mod indicator functions of cones with lines } .
$$

From Proposition 2.19 and the valuation property of intermediate generating functions, we obtain Brion's formula (cf. [8]), which expresses the holomorphic function $S^{L}(\mathfrak{p}(b))(\xi)$ as a sum of meromorphic functions indexed by the vertices of the polytope $\mathfrak{p}(b)$.

$$
S^{L}(\mathfrak{p}(b))(\xi)=\sum_{B \in \mathcal{B}_{\tau}} S^{L}\left(s_{B}(b)+\mathfrak{c}_{B}\right)(\xi), \text { for } b \in \bar{\tau} .
$$

2.4. SteP-POlynOmials AND (SEMI-)QUASI-POLYNOMIALS OF THE MULTI-PARAMETER $b$. In this section, we consider a fixed rational subspace $L \subseteq V$. There corresponds an intermediate generating function $S^{L}(\mathfrak{p}(b))(\xi)$ and intermediate weighted sums $S^{L}(\mathfrak{p}(b), h)$ on a parametric polytope $\mathfrak{p}(b)$.

Recall from the introduction the algebras of step-polynomials and quasi-polynomials on $\mathbb{R}^{N}$, in terms of which we will describe these intermediate sums as functions of the parameter $b \in \mathbb{R}^{N}$. We give more general definitions now.

Definition 2.20. Let $\Psi \subseteq \mathbb{R}^{N}$.

(1) $\mathcal{Q}^{\Psi}\left(\mathbb{R}^{N}\right)$ is the algebra of functions on $\mathbb{R}^{N}$ generated by the functions $b \mapsto$ $\{\langle\eta, b\rangle\}$, where $\eta \in \Psi$. An element of $\mathcal{Q}^{\Psi}\left(\mathbb{R}^{N}\right)$ is called a step-polynomial on $\mathbb{R}^{N}$.

(2) For $\Psi=\mathbb{Q}^{N}$, we obtain the algebra of rational step-polynomials, which we abbreviate as $\mathcal{Q}\left(\mathbb{R}^{N}\right)$.

(3) For $\Psi=\mathbb{R}^{N}$, we obtain the algebra of step-polynomials $\mathcal{Q}^{\mathbb{R}^{N}}\left(\mathbb{R}^{N}\right)$.

Note that a step-polynomial function (whether rational or not) is a bounded function on $\mathbb{R}^{N}$.

The algebra $\mathcal{Q}^{\Psi}\left(\mathbb{R}^{N}\right)$ has a natural filtration, where $\mathcal{Q}_{[\leqslant k]}^{\Psi}\left(\mathbb{R}^{N}\right)$ is the subspace spanned by products of at most $k$ functions $\{\langle\eta, b\rangle\}$. Again this is a filtration, not a grading, because several step-polynomials with different (step) degrees may represent the same function.

EXAMPLE 2.21. For every $t \in \mathbb{R}$,

$$
1-\{t\}-\{-t\}-(1-\{2 t\}-\{-2 t\})(1-\{3 t\}-\{-3 t\})=0 .
$$

Definition 2.22. Again let $\Psi \subseteq \mathbb{R}^{N}$.

(1) The tensor product $\mathcal{Q} \mathcal{P}^{\Psi}\left(\mathbb{R}^{N}\right)=\mathcal{Q}^{\Psi}\left(\mathbb{R}^{N}\right) \otimes \mathcal{P}\left(\mathbb{R}^{N}\right)$ is the algebra of functions on $\mathbb{R}^{N}$ generated by step-polynomials in $\mathcal{Q}^{\Psi}\left(\mathbb{R}^{N}\right)$ and ordinary polynomials on $\mathbb{R}^{N}$. An element of $\mathcal{Q} \mathcal{P}^{\Psi}\left(\mathbb{R}^{N}\right)$ is called a semi-quasi-polynomial on $\mathbb{R}^{N}$.

(2) For $\Psi=\mathbb{Q}^{N}$, we obtain the algebra of quasi-polynomials on $\mathbb{R}^{N}$, which we abbreviate as $\mathcal{Q P}\left(\mathbb{R}^{N}\right)$.

(3) For $\Psi=\mathbb{R}^{N}$, we obtain the algebra of semi-quasi-polynomials on $\mathbb{R}^{N}$, denoted by $\mathcal{Q} \mathcal{P}^{N}\left(\mathbb{R}^{N}\right)$. 
A (semi-)quasi-polynomial $f(b)$ is a piecewise polynomial. More precisely, let $\Psi$ be a finite set of $\eta \in \mathbb{R}^{N}$ such that $f \in \mathcal{Q}^{\Psi}\left(\mathbb{R}^{N}\right)$, i.e., $f(b)$ can be expressed as a polynomial in the functions $b \mapsto\{\langle\eta, b\rangle\}$, with $\eta \in \Psi$. The open "pieces" on which $f(b)$ is a polynomial function of $b$ are the $\Psi$-alcoves defined as follows.

DEFINITION 2.23. Let $\Psi$ be a finite subset of $\mathbb{R}^{N}$. We consider the hyperplanes in $\mathbb{R}^{N}$ defined by the equations

$$
\langle\eta, b\rangle=n, \text { for } \eta \in \Psi \text { and } n \in \mathbb{Z} .
$$

A connected component of the complement of the union of these hyperplanes is called a $\Psi$-alcove.

On the tensor product $\mathcal{Q} \mathcal{P}^{\Psi}\left(\mathbb{R}^{N}\right)$, we will consider the grading inherited from the usual degree on $\mathcal{P}\left(\mathbb{R}^{N}\right)$. We will call the corresponding degree the polynomial degree.

We consider also the degree arising from the tensor product filtration, which we call the local degree. With these notations, if $f(b) \in \mathcal{Q} \mathcal{P}_{[\leqslant k]}^{\Psi}\left(\mathbb{R}^{N}\right)$, then $f(b)$ restricts as a polynomial function of degree $\leqslant k$ on any $\Psi$-alcove.

EXAMPLE 2.24. The quasi-polynomial $f(b)=b^{3}\{b\}$ on $\mathbb{R}$ has polynomial degree 3 and local degree 4 . It is equal to $b^{4}-n b^{3}$, a polynomial of degree 4 , for $n \leqslant b<n+1$.

In the remainder of this section, we will have $\Psi \subset \mathbb{Q}^{N}$, and hence work with rational step-polynomials and quasi-polynomials; however, in Subsection 2.5, we will use more general $\Psi$.

We now explain how to construct a finite set $\Psi$ that is suitable for our multiparameter Ehrhart quasi-polynomials.

In [4], for any rational cone $\mathfrak{c} \subset V$, and a rational subspace $L$, we constructed a finite subset $\Psi_{\mathfrak{c}}^{L} \subset \Lambda^{*} \cap L^{\perp}$ of integral linear forms on $V$ [4, Definition 2.24]. We briefly recall the steps of this construction; the details can be found in [4, proof of Lemma 2.10]. We start by decomposing $\mathfrak{c}$ in cones $\mathfrak{u}$ (modulo cones with lines) with a face parallel to $L$, then we decompose the projections of $\mathfrak{u}$ on $V / L$ as a signed decomposition of unimodular cones with respect to the projected lattice $\Lambda_{V / L}$ with dual lattice $\Lambda^{*} \cap L^{\perp}$. In the set $\Psi_{\mathfrak{c}}^{L}$, we collect all the generators of the dual cones used in this decomposition. (The decomposition is not unique, but we do not record the dependence of $\Psi_{\mathfrak{c}}^{L}$ on the decomposition in the notation.) As $\Psi_{\mathfrak{c}}^{L} \subset \Lambda^{*} \cap L^{\perp}$, the step-polynomials in the algebra $\mathcal{Q}^{\Psi_{\mathrm{c}}^{L}}(V)$ (see Subsection 2.2) are functions on $V /(\Lambda+L)$.

\section{DEFINITION 2.25}

(1) Let $B \in \mathcal{B}$. Define $\Psi_{B}^{L}(\alpha)$ as the set of rational linear forms on $\mathbb{R}^{N}$ defined by $\langle\eta, b\rangle=\left\langle\gamma, s_{B}(b)\right\rangle$, for $\gamma \in \Psi_{\mathfrak{c}_{B}}^{L}$.

(2) If $\tau$ is an $\alpha$-chamber, $\Psi_{\tau}^{L}(\alpha)$ is the union of the sets $\Psi_{B}^{L}(\alpha)$ when $B$ runs in $\mathcal{B}_{\tau}$.

By definition, if $f \in \mathcal{Q} \mathcal{P}^{\Psi_{\mathbf{c}_{B}}^{L}}(V)$ (see Subsection 2.2), then the function $b \mapsto$ $f\left(s_{B}(b)\right)$ is in $\mathcal{Q} \mathcal{P}^{\Psi_{B}^{L}(\alpha)}\left(\mathbb{R}^{N}\right)$.

Let us describe a little more precisely the sets $\Psi_{\mathfrak{c}_{B}}^{L}$ and the corresponding steppolynomials on $\mathbb{R}^{N}$ when $L=V$ or $L=\{0\}$.

When $L=V$, the sets $\Psi_{\mathfrak{c}_{B}}^{L}$ are empty, thus the step-polynomials in $\mathcal{Q}^{\Psi_{\tau}^{L}(\alpha)}\left(\mathbb{R}^{N}\right)$ are just the constants.

When $L=\{0\}$, let $q \in \mathbb{N}$ be such that the lattice $q \Lambda^{*}$ is contained in the lattice generated by the elements $\alpha_{j}$, for $j \in B$. Then the multiple $q s_{B}(b)$ of the vertex $s_{B}(b)$ belongs to $\Lambda$ if $b \in \mathbb{Z}^{N}$, therefore any step-polynomial $f(b) \in \mathcal{Q}^{\Psi_{B}^{\{0\}}(\alpha)}\left(\mathbb{R}^{N}\right)$ is 
$q \mathbb{Z}^{N}$-periodic. Thus a quasi-polynomial $f$ on $\mathbb{R}^{N}$ gives by restriction to $\mathbb{Z}^{N}$ a periodic function of period $q$, and we recover the usual notion of quasi-periodic function on a lattice.

If $\mathfrak{c}_{B}$ is the simplicial cone described by inequalities $\left\langle\alpha_{j}, x\right\rangle \leqslant 0$, with $j \in B$, and if the $\alpha_{j}, j \in B$ form a basis of $\Lambda^{*}$, then the set $\Psi_{\mathfrak{c}_{B}}^{\{0\}} \subset \Lambda^{*}$ is just equal to $\left\{\alpha_{j}: j \in B\right\}$. Thus if the sequence $\alpha$ is unimodular, that is, if $\left\{\alpha_{j}: j \in B\right\}$ is a basis of $\Lambda^{*}$ for any $B \in \mathcal{B}$, any step-polynomial $f(b) \in \mathcal{Q}^{\Psi_{\tau}^{L}(\alpha)}\left(\mathbb{R}^{N}\right)$ is $\mathbb{Z}^{N}$-periodic, in particular $f(b)$ is constant on $\mathbb{Z}^{N}$.

\subsection{Weighted Ehrhart (SEMI-)QUASI-POLYNOMIALS.}

2.5.1. Case of a parametric polytope. We can now state the first important result of this article. We summarized it in the introduction, in a less technical form, as Theorem 1.1.

TheOREM 2.26. Let $V$ be a rational vector space with lattice $\Lambda$. Let $L \subseteq V$ be a rational subspace. Let $\alpha=\left(\alpha_{1}, \ldots, \alpha_{N}\right)$ be a list of elements of $\Lambda^{*}$ which generate $V^{*}$ as a cone. For $b \in \mathbb{R}^{N}$, let $\mathfrak{p}(b) \subset V$ be the polytope defined by

$$
\mathfrak{p}(b)=\left\{x \in V:\left\langle\alpha_{j}, x\right\rangle \leqslant b_{j}, j=1, \ldots, N\right\} .
$$

Let $\tau \subset \mathbb{R}^{N}$ be an admissible $\alpha$-chamber. Let $h$ be a polynomial function on $V$ of degree $m$.

(1) There exists a quasi-polynomial of local degree equal to $m+d$,

$$
E^{L}(\alpha, h, \tau) \in \mathcal{Q} \mathcal{P}_{[\leqslant m+d]}^{\Psi_{\tau}^{L}(\alpha)}\left(\mathbb{R}^{N}\right)
$$

such that

$$
S^{L}(\mathfrak{p}(b), h)=E^{L}(\alpha, h, \tau)(b),
$$

for every $b \in \bar{\tau}$ (the closure of $\tau$ in $\mathbb{R}^{N}$ ).

(2) If $h(x)$ is homogeneous of degree $m$, then the terms of $E^{L}(\alpha, h, \tau)(b)$ of polynomial degree $m+d$ form a homogeneous polynomial of degree $m+d$ that is equal to the integral $\int_{\mathfrak{p}(b)} h(x) \mathrm{d} x$ for $b \in \bar{\tau}$.

(3) More precisely, if $h(x)=\frac{\langle\ell, x\rangle^{m}}{m !}$ for some $\ell \in V^{*}$, we have

$$
E^{L}(\alpha, h, \tau)(b)=\sum_{r=0}^{m+d} E_{[r]}^{L}(\alpha, h, \tau)(b)
$$

for $b \in \bar{\tau}$, where for each $r$, the function of $b \in \mathbb{R}^{N}$ given by

$$
E_{[r]}^{L}(\alpha, h, \tau)(b)=\left.\left(\sum_{B \in \mathcal{B}_{\tau}} M^{L}\left(s_{B}(b), \mathfrak{c}_{B}\right)_{[m-r]}(\xi) \frac{\left\langle\xi, s_{B}(b)\right\rangle^{r}}{r !}\right)\right|_{\xi=\ell}
$$

is an element of $\mathcal{Q}_{[\leqslant m+d-r]}^{\Psi_{\tau}^{L}(\alpha)}\left(\mathbb{R}^{N}\right) \otimes \mathcal{P}_{[r]}\left(\mathbb{R}^{N}\right)$, i.e., of polynomial degree $r$ and local degree at most $m+d$.

In fact, for a single $B, M^{L}\left(s_{B}(b), \mathfrak{c}_{B}\right)_{[m-r]}$ is a rational function of $\xi$ and may be singular at $\ell$, so that the value $M^{L}\left(s_{B}(b), \mathfrak{c}_{B}\right)_{[m-r]}(\ell)$ may not be well defined. However, as we will see in the proof, for each $r$, the sum over the $B \in \mathcal{B}_{\tau}$ of the rational functions $M^{L}\left(s_{B}(b), \mathfrak{c}_{B}\right)_{[m-r]}(\xi) \frac{\left\langle\xi, s_{B}(b)\right\rangle^{r}}{r !}$ is a polynomial function of $\xi$, so it can be evaluated at $\xi=\ell$. So Formula (18) is well defined. 
Proof. The proof of Theorem 2.26 rests on Brion's formula (15). We observe that it is enough to prove the theorem in the case where the weight $h$ is a power of a linear form,

$$
h(x)=\frac{\langle\ell, x\rangle^{m}}{m !}
$$

for some $\ell \in V^{*}$, as any weight can be written as a linear combination of those. In this case, $S^{L}(\mathfrak{p}(b), h)$ is the value at $\xi=\ell$ of the homogeneous term of degree $m$ of the holomorphic function $S^{L}(\mathfrak{p}(b))(\xi)$. So we write (using the fundamental Equation (9))

$$
\begin{aligned}
S^{L}(\mathfrak{p}(b), h)_{[m]}(\ell) & =\left.\left(\sum_{B \in \mathcal{B}_{\tau}} S^{L}\left(s_{B}(b)+\mathfrak{c}_{B}\right)_{[m]}(\xi)\right)\right|_{\xi=\ell} \\
& =\left.\sum_{r=0}^{m+d}\left(\sum_{B \in \mathcal{B}_{\tau}} M^{L}\left(s_{B}(b), \mathfrak{c}_{B}\right)_{[m-r]}(\xi) \frac{\left\langle\xi, s_{B}(b)\right\rangle^{r}}{r !}\right)\right|_{\xi=\ell}
\end{aligned}
$$

For an individual $B$, the term $S^{L}\left(s_{B}(b)+\mathfrak{c}_{B}\right)_{[m]}(\xi)$ may be singular at $\xi=\ell$. However the sum over the set of vertices $\mathcal{B}_{\tau}$ is a polynomial function of $\xi$.

In [4] we studied the bidegree structure of $M^{L}(s, \mathfrak{c})(\xi)$, i.e., the interaction of the local degree in $s$ and the homogeneous degree in $\xi$, which allows us to extract the refined asymptotics. For each $r$ and $B$, we consider the function of $s \in V, \xi \in V^{*}$, given by $(s, \xi) \mapsto M^{L}\left(s, \mathfrak{c}_{B}\right)_{[m-r]}(\xi)$. By Theorem 2.25 of [4], this function belongs to the space

$$
\mathcal{Q}_{[\leqslant m+d-r]}^{\Psi_{\mathbf{c}_{B}}^{L}}(V) \otimes \mathcal{R}_{[m-r]}\left(V^{*}\right) .
$$

Compose with the linear map $b \mapsto s_{B}(b)$. We obtain that, for each $r$ and $B$, the function of $b \in \mathbb{R}^{N}, \xi \in V^{*}$ given by

belongs to

$$
(b, \xi) \mapsto M^{L}\left(s_{B}(b), \mathfrak{c}_{B}\right)_{[m-r]}(\xi) \frac{\left\langle\xi, s_{B}(b)\right\rangle^{r}}{r !}
$$

$$
\mathcal{Q}_{[\leqslant m+d-r]}^{\Psi_{B}^{L}(\alpha)}\left(\mathbb{R}^{N}\right) \otimes \mathcal{P}_{[r]}\left(\mathbb{R}^{N}\right) \otimes \mathcal{R}_{[m]}\left(V^{*}\right)
$$

Therefore the sum over $B \in \mathcal{B}_{\tau}$ of these terms, for a fixed $r$, belongs to

$$
\mathcal{Q}_{[\leqslant m+d-r]}^{\Psi_{\tau}^{L}(\alpha)}\left(\mathbb{R}^{N}\right) \otimes \mathcal{P}_{[r]}\left(\mathbb{R}^{N}\right) \otimes \mathcal{R}_{[m]}\left(V^{*}\right)
$$

Now the sum over $B \in \mathcal{B}_{\tau}$ is a quasi-polynomial function of $b$ with values in the space of polynomials in $\xi$, not just rational functions of $\xi$. It follows that for each $r$, the term of polynomial degree $r$ in $b$ of the full sum depends also polynomially on $\xi$. This term is

$$
\sum_{B \in \mathcal{B}_{\tau}} M^{L}\left(s_{B}(b), \mathfrak{c}_{B}\right)_{[m-r]}(\xi) \frac{\left\langle\xi, s_{B}(b)\right\rangle^{r}}{r !} .
$$

When we evaluate it at $\xi=\ell$, we obtain (18), and all statements but part (2), which we prove now.

Let us compute the term of polynomial degree $r=m+d$ with respect to $b$, in (18). From Equation (10), we know that $M^{L}\left(s_{B}(b), \mathfrak{c}_{B}\right)_{[-d]}(\xi)=I\left(\mathfrak{c}_{B}\right)(\xi)$, thus the term of index $r=m+d$ in (18) is equal to

$$
\left.\left(\sum_{B \in \mathcal{B}_{\tau}} \frac{\left\langle\xi, s_{B}(b)\right\rangle^{m+d}}{(m+d) !} I\left(\mathfrak{c}_{B}\right)(\xi)\right)\right|_{\xi=\ell}=\left.\left(\sum_{B \in \mathcal{B}_{\tau}} I\left(s_{B}(b)+\mathfrak{c}_{B}\right)_{[m]}(\xi)\right)\right|_{\xi=\ell} .
$$

By Proposition 2.19, this last sum is equal to $I(\mathfrak{p}(b))_{[m]}(\ell)$, which is precisely the integral $\int_{\mathfrak{p}(b)} \frac{\langle\ell, x\rangle^{m}}{m !} \mathrm{d} x$. 
Definition 2.27. The function $E^{L}(\alpha, h, \tau)(b)$ of Theorem 2.26 is called the weighted intermediate Ehrhart quasi-polynomial of the parametric polytope $\mathfrak{p}(b)$ (with respect to the weight $h$, the subspace $L$ and the chamber $\tau$ ).

EXAMPle 2.28 (Example 2.7, continued). Let $\alpha=(x,-x)$. Then for $b=\left(b_{1}, b_{2}\right) \in \mathbb{R}^{2}$, $\mathfrak{p}(b)$ is the interval $\left\{x:-b_{2} \leqslant x \leqslant b_{1}\right\}$. There are two chambers, $\left\{b:-b_{2}<\right.$ $\left.b_{1}\right\}$ and $\left\{b:-b_{2}>b_{1}\right\}$. For the first chamber the number of integers in $\mathfrak{p}(b)$ is $\left\lfloor b_{1}\right\rfloor-\left\lceil-b_{2}\right\rceil+1=b_{1}+b_{2}-\left\{b_{1}\right\}-\left\{b_{2}\right\}+1$. For the other chamber, it is of course 0 .

EXAMPLE 2.29 (continuation of Examples 2.8, 2.10, and 2.15). We compute the quasipolynomial function $E^{L}\left(\alpha, h, \tau_{2}\right)(b)\left(\mathfrak{p}(b)\right.$ is a quadrilateral for $\left.b \in \tau_{2}\right)$, first for $L=$ $\{0\}$, then for the case when $L$ is the vertical line $L=\mathbb{R}(0,1)$. The weight is $h(x)=1$.

(1) For $L=\{0\}$ and $h=1$, i.e., we count the integer points in $\mathfrak{p}(b)$, we write

$$
E^{\{0\}}\left(\alpha, 1, \tau_{2}\right)(b)=E_{[2]}(b)+E_{[1]}(b)+E_{[0]}(b),
$$

where $E_{[r]}(b)$ collects the terms of polynomial degree $r$ with respect to $b$. $E_{[2]}(b)$ is the volume of the quadrilateral. It is a polynomial function, easy to compute. The other two functions were computed with our Maple program.

$$
\begin{aligned}
& E_{[2]}(b)=- \frac{b_{1}^{2}}{2}+\frac{b_{2}^{2}}{2}+\frac{b_{3}^{2}}{4}-\frac{b_{4}^{2}}{4}+b_{1} b_{2}+b_{1} b_{4}+b_{2} b_{3}+\frac{b_{3} b_{4}}{2} . \\
& E_{[1]}(b)=\left(\frac{1}{2}+\left\{b_{1}\right\}-\left\{b_{2}\right\}-\left\{b_{4}\right\}\right) b_{1} \\
&+\left(\frac{3}{2}-\left\{b_{1}\right\}-\left\{b_{2}\right\}-\left\{b_{3}\right\}\right) b_{2} \\
&+\left(1-\left\{b_{2}\right\}-\frac{1}{2}\left\{b_{3}\right\}-\frac{1}{2}\left\{b_{4}\right\}\right) b_{3} \\
&+\left(\frac{1}{2}-\left\{b_{1}\right\}-\frac{\left\{b_{3}\right\}}{2}+\frac{\left\{b_{4}\right\}}{2}\right) b_{4} . \\
& E_{[0]}(b)=1-\frac{1}{2}\left\{b_{1}\right\}-\frac{3}{2}\left\{b_{2}\right\}-\left\{b_{3}\right\}-\frac{1}{2}\left\{b_{4}\right\} \\
&-\frac{1}{2}\left\{b_{1}\right\}^{2}+\frac{1}{2}\left\{b_{2}\right\}^{2}-\frac{1}{2}\left\{b_{4}\right\}^{2}-\left\{\frac{b_{4}+b_{3}}{2}\right\}^{2} \\
&+\left\{b_{1}\right\}\left\{b_{2}\right\}+\left\{b_{1}\right\}\left\{b_{4}\right\}+\left\{b_{2}\right\}\left\{b_{3}\right\} \\
&+\left\{b_{3}\right\}\left\{\frac{b_{4}+b_{3}}{2}\right\}+\left\{b_{4}\right\}\left\{\frac{b_{4}+b_{3}}{2}\right\} .
\end{aligned}
$$

We see that $E_{[2]}(b)$ is a linear combination of products of two linear forms, $E_{[1]}(b)$ is a linear combination of products of linear forms with step-linear forms, while $E_{[0]}(b)$ is a linear combination of products of at most two steplinear forms. Thus each of the $E_{[r]}(b)$ is of local degree 2 .

(2) We compute the intermediate quasi-polynomial

$$
E^{L}\left(\alpha, 1, \tau_{2}\right)(b)=E_{[2]}^{L}(b)+E_{[1]}^{L}(b)+E_{[0]}^{L}(b)
$$

for the same chamber $\tau_{2}$, when $L$ is the vertical line $L=\mathbb{R}(0,1)$, and again $h=$ 1. Thus we compute the sum $S^{L}(\mathfrak{p}(b), 1)$ of the lengths of vertical segments in the quadrilateral $\mathfrak{p}(b)$. Then $E_{[2]}^{L}(b)=E_{[2]}(b)=\operatorname{vol}(\mathfrak{p}(b))$ is again the volume 
of $\mathfrak{p}(b)$, and we compute

$$
\begin{aligned}
E_{[1]}^{L}(b)= & -\frac{1}{2} b_{1}+\frac{1}{2} b_{2}+\frac{1}{2} b_{4}+\left\{b_{1}\right\} b_{1}-\left\{b_{1}\right\} b_{2}-\left\{b_{1}\right\} b_{4}, \\
E_{[0]}^{L}(b)= & \frac{1}{2}\left\{b_{1}\right\}+\frac{1}{2}\left\{b_{2}+b_{3}\right\}-\left\{\frac{b_{3}-b_{4}}{2}\right\} \\
& -\frac{1}{2}\left\{b_{1}\right\}^{2}-\frac{1}{2}\left\{b_{2}+b_{3}\right\}^{2}+\left\{\frac{b_{3}-b_{4}}{2}\right\}^{2} .
\end{aligned}
$$

Again, we observe that the local degree of $E_{[r]}^{L}(b)$ is indeed 2 for $r=0,1,2$.

REMARK 2.30. In this theorem, the parameter $b$ varies in $\mathbb{R}^{N}$. In particular, the results of [14] on "vector dilated polytopes" follow easily from this theorem. ${ }^{(2)}$ The article [14] was part of our motivation to consider the case of a multidimensional real-valued parameter and not just one parameter dilations.

REMARK 2.31. When $L=V$, we are computing an integral over $\mathfrak{p}(b)$. It is clearly a polynomial function of $b$ on any chamber. This is consistent with the fact that $\mathcal{Q}^{\Psi_{\tau}^{V}(\alpha)}\left(\mathbb{R}^{N}\right)$ is just the constants.

Classically, in particular when computing the number of lattice points (case $L=$ $\{0\}, h(x)=1$ ), the parameter $b$ was restricted to $\mathbb{Z}^{N}$. As we already observed, if $q \Lambda^{*}$ is contained in the lattice generated by $\left(\alpha_{j}, j \in B\right)$ for any $B \in \mathcal{B}_{\tau}$, then the coefficients of the Ehrhart quasi-polynomial (16) are $q \mathbb{Z}^{N}$-periodic functions on $\mathbb{R}^{N}$, therefore the Ehrhart quasi-polynomial restricts to any coset $\left\{b_{0}+q n: n \in \mathbb{Z}^{N}\right\}$ as a true polynomial function of $n \in \mathbb{Z}^{N}$.

REMARK 2.32 (Case of partition polytopes). The paper [10] deals with Ehrhart quasipolynomials for weighted sums and integrals over a partition polytope. Their variation is computed, when the parameter crosses a wall between two chambers. Let us recall how a parametric polytope $\mathfrak{p}(\alpha, b)$ is associated to a partition polytope $\mathfrak{p}^{\text {partition }}(\Phi, \lambda)$. Let $F$ be a vector space of dimension $N-d$ and let $\Phi=\left(\phi_{1}, \ldots, \phi_{N}\right)$ be a sequence of elements of $F$. We assume that $\Phi$ generates a full-dimensional pointed cone in $F$. For $\lambda \in F$, let

$$
\mathfrak{p}^{\text {partition }}(\Phi, \lambda)=\left\{y=\left(y_{j}\right) \in \mathbb{R}^{N}: y_{j} \geqslant 0, \sum_{j=1}^{N} y_{j} \phi_{j}=\lambda\right\} .
$$

This is a polytope contained in the affine subspace $\left\{y=\left(y_{j}\right) \in \mathbb{R}^{N}: \sum_{j=1}^{N} y_{j} \phi_{j}=\lambda\right\}$. Define $V:=\left\{y=\left(y_{j}\right) \in \mathbb{R}^{N}: \sum_{j=1}^{N} y_{j} \phi_{j}=0\right\}$. Let $\alpha_{j}$ be the linear form on $V$ defined by $\left\langle\alpha_{j}, x\right\rangle=-x_{j}$. For $b \in \mathbb{R}^{N}$, let $\lambda=\sum_{j=1}^{N} b_{j} \phi_{j}$. Then $x \mapsto x+b$ is a bijection between $\mathfrak{p}(\alpha, b)$ and $\mathfrak{p}^{\text {partition }}(\Phi, \lambda)$.

REMARK 2.33 (Wall crossing). Finally, it would be interesting to study the variation of the quasi-polynomials $S^{L}(\mathfrak{p}(b), h)$ when $b$ crosses the wall of a chamber $\tau$. The method of [10] could probably be adapted to the more general case of intermediate weighted sums of a parametric polytope.

2.5.2. Specialization to other parameter domains. From the study of a general parametric polytope, it is not difficult to derive results when the multi-parameter $b \in \mathbb{R}^{N}$ is itself a function of another parameter $t \in \mathbb{R}^{q}, b=b\left(t_{1}, \ldots, t_{q}\right)$. We restrict ourselves to the setting where $b$ is a (homogeneous) linear function of $t$, which we write as $b(t)=T t$, where $T \in \mathbb{R}^{N \times q}$ is a matrix. This is sufficient for two popular settings, which we explain in the following sections. In Section 2.5.3, we will consider the case

\footnotetext{
${ }^{(2)}$ Note that [14] states and proves results for rational vector dilations only.
} 

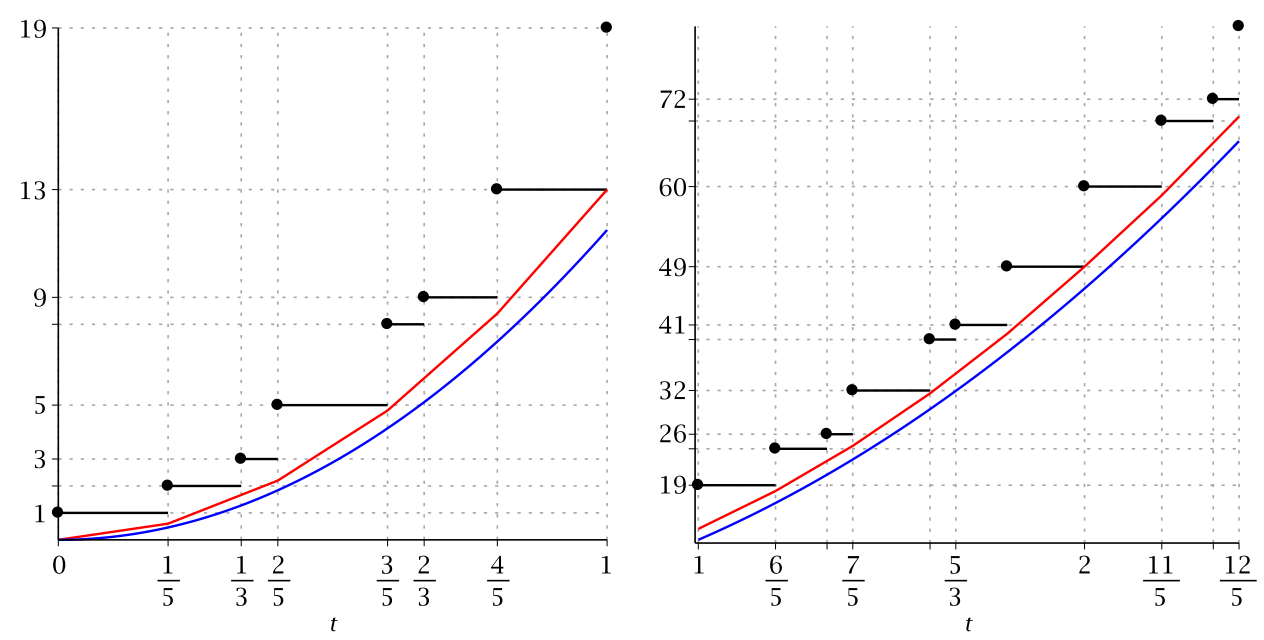

FiguRE 8. $S^{L}(t \mathfrak{p}, 1)$ for the quadrilateral of Example 2.34, for $L=$ $\{0\}$ (black), $L$ vertical (red), $L=\mathbb{R}^{2}$ (blue). On the left, $t$ varies from 0 to 1 , new lattice points occur for $t=0, \frac{1}{5}, \frac{1}{3}, \frac{2}{5}, \frac{3}{5}, \frac{2}{3}, \frac{4}{5}, 1$. On the right, $t$ varies from 1 to 2.4 , new lattice points occur for $t=\frac{6}{5}, \frac{4}{3}, \frac{7}{5}, \frac{8}{5}, \frac{5}{3}, 2, \frac{11}{5}, \frac{7}{3}, \frac{12}{5}$. For $L$ vertical, $S^{L}(t \mathfrak{p}, 1)$ is continuous, but its derivative has discontinuities.

of a fixed semi-rational polytope $\mathfrak{p}$ dilated by a one-dimensional real parameter $t \geqslant 0$. In Section 2.5.4, we will consider the more general case of a Minkowski linear system $t_{1} \mathfrak{p}_{1}+\cdots+t_{q} \mathfrak{p}_{q}$.

To describe how the specialization yields the function $t \mapsto E^{L}(\alpha, h, \tau)(T t)$, let us first describe the alcoves. Let $T^{*} \in \mathbb{R}^{q \times N}$ be the adjoint (transpose) matrix. The linear forms $\eta \in \Psi_{\tau}^{L}(\alpha) \subset \mathbb{Q}^{N}$ defining the alcoves of $\mathbb{R}^{N}$ (see Theorem 2.26) give rise to linear forms on $\mathbb{R}^{q}$,

$$
\left\langle T^{*} \eta, t\right\rangle=\langle\eta, T t\rangle \quad \text { for } t \in \mathbb{R}^{q} .
$$

Thus we consider the alcoves of $\mathbb{R}^{q}$ defined by the finite set $T^{*}\left(\Psi_{\tau}^{L}(\alpha)\right) \subset \mathbb{R}^{q}$. Note that, when $T$ is not rational, $T^{*}\left(\Psi_{\tau}^{L}(\alpha)\right)$ will no longer be rational, in contrast to the development in Subsection 2.4. The function $t \mapsto E^{L}(\alpha, h, \tau)(T t)$ will therefore belong to the subalgebra $\mathcal{Q} \mathcal{P}^{T^{*}\left(\Psi_{\tau}^{L}(\alpha)\right)}\left(\mathbb{R}^{q}\right)$ of semi-quasi-polynomials. (When $T$ is rational, this is a subalgebra of quasi-polynomials.)

Using this notation, we can formulate a theorem analogous to Theorem 2.26. We omit the statement.

In contrast to Theorem 2.26, we no longer know the precise local degree of the semiquasi-polynomial $t \mapsto E^{L}(\alpha, h, \tau)(T t)$. The "expected" degree is $m+d$, but there may be cancellations of terms, as illustrated by the example $\mathfrak{p}=[-1,1], h(x)=x$ given in the introduction.

2.5.3. Case of a dilated polytope. A first example appeared in the introduction as Example 1.4, which already illustrated that in the case of semi-rational polytopes $\mathfrak{p}$ which are not rational, we may not get quasi-polynomials of the dilation factor $t$ but merely semi-quasi-polynomials. Let us give a few more examples for the rational case.

ExAmple 2.34 (Continuation of Examples 2.8, 2.10 and 2.15). Fix $b_{0}=(0,0,5,3)$, so that $\mathfrak{p}=\mathfrak{p}\left(b_{0}\right)$ is the quadrilateral of Figure 5 with vertices $[0,0],[0,3],[1,4],[5,0]$. We 
specialize the formula for $E^{L}\left(\alpha, 1, \tau_{2}\right)(b)$ to the line $b=t b_{0}$. We consider the cases $L=\{0\}, L$ the vertical line, and $L=V$.

(1) First, with $L=V$, we compute the volume. For $t \geqslant 0, S^{V}(t \mathfrak{p})=\frac{23}{2} t^{2}$. It is a polynomial function of $t$ with rational coefficients.

(2) Next, with $L=\{0\}$, we count the lattice points of $t \mathfrak{p}$, for $t \geqslant 0$.

$$
\begin{aligned}
S^{\{0\}}(t \mathfrak{p}, 1)=\frac{23}{2} & t^{2}+\left(\frac{13}{2}-\{3 t\}-4\{5 t\}\right) t \\
& -\frac{1}{2}\{3 t\}^{2}-\{4 t\}^{2}+\{4 t\}\{3 t\}+\{5 t\}\{4 t\} \\
& -\{5 t\}-\frac{1}{2}\{3 t\}+1 .
\end{aligned}
$$

It takes only integral values, and is locally constant over some rational intervals. These facts are more apparent on the graph (Figure 8) than on the formula. When $t$ is in $\mathbb{Z}$, all terms $\{q t\}$, for $q \in \mathbb{Z}$, are equal to 0 , and we obtain the usual Ehrhart polynomial of $\mathfrak{p}$ over $\mathbb{Z}$ (it is a polynomial as $\mathfrak{p}$ has integral vertices)

$$
\frac{23}{2} t^{2}+\frac{13}{2} t+1
$$

The value at $t=1$ is 19 , the number of integral points in $\mathfrak{p}$.

(3) When $L$ is the vertical line, we add the lengths of the vertical segments in $t \mathfrak{p}$, for $t \geqslant 0$.

$$
S^{L}(t \mathfrak{p}, 1)=\frac{23}{2} t^{2}+\frac{3}{2} t+\frac{1}{2}\{5 t\}^{2}+\{t\}^{2}-\{4 t\}^{2}+\frac{1}{2}\{5 t\}-\{t\} .
$$

This is a continuous function of $t$. Its value at $t=1$ is 13 .

Now we describe how this specialization works in general. Let $L$ be a rational subspace of $V$. We take a (semi-)rational polytope $\mathfrak{p}=\mathfrak{p}\left(b_{0}\right)$ associated to a fixed real multi-parameter $b_{0}$, and specialize the formula for $E^{L}(\alpha, h, \tau)(b)$, where $b_{0} \in \bar{\tau}$, when $b=t b_{0}$ for $t \in \mathbb{R}, t>0$. Using the notation from Subsubsection 2.5.2, we have the matrix $T=\left(b_{0}\right) \in \mathbb{R}^{N \times 1}$. We then compute the finite set $\Psi:=T^{*}\left(\Psi_{\tau}^{L}(\alpha)\right) \subset \mathbb{R}$. It can be described in a simpler way as follows. Denote by $\Psi_{\mathfrak{p}}^{L}$ the union of the sets $\Psi_{\mathfrak{c}}^{L} \subset \Lambda^{*} \cap L^{\perp}$, described in Subsection 2.4, where $\mathfrak{c}$ varies over the cones of feasible directions at the vertices of $\mathfrak{p}$ (they are rational polyhedral cones).

Then $\Psi$ is the finite set of real numbers $\langle\gamma, s\rangle$, where $\gamma$ runs in $\Psi_{\mathfrak{p}}^{L}$, and $s$ runs over the vertices of $\mathfrak{p}$. It describes the alcoves of $\mathbb{R}$. Thus the function $t \mapsto S^{L}(t \mathfrak{p}, h)$ is a (semi-)quasi-polynomial, which coincides with a polynomial function of $t$ on intervals with possibly irrational ends, as in Example 1.4. Its coefficient functions are bounded functions of the variable $t \in \mathbb{R}$. If $\mathfrak{p}$ is rational, then $\Psi$ is rational, and thus the coefficient functions are periodic functions.

We summarize this discussion in the following result.

THEOREM 2.35. Let $\mathfrak{p}$ be a (semi-)rational polytope and $h$ a polynomial function of degree $m$ on $V$. Let $\Psi \subset \mathbb{R}$ be the set of real numbers $\langle\gamma, s\rangle$, where $\gamma$ runs in $\Psi_{\mathfrak{p}}^{L}$ and $s$ runs over the vertices of $\mathfrak{p}$.

(1) There exists a (semi-)quasi-polynomial

$$
E^{L}(\mathfrak{p}, h)(t)=\sum_{r=0}^{d+m} E_{r}^{L}(\mathfrak{p}, h)(t) t^{r},
$$

such that $S^{L}(t \mathfrak{p}, h)=E^{L}(\mathfrak{p}, h)(t)$ for all $t \in \mathbb{R}$ with $t \geqslant 0$. It belongs to $\mathcal{Q P}_{[\leqslant m+d]}^{\Psi}(\mathbb{R})$. 

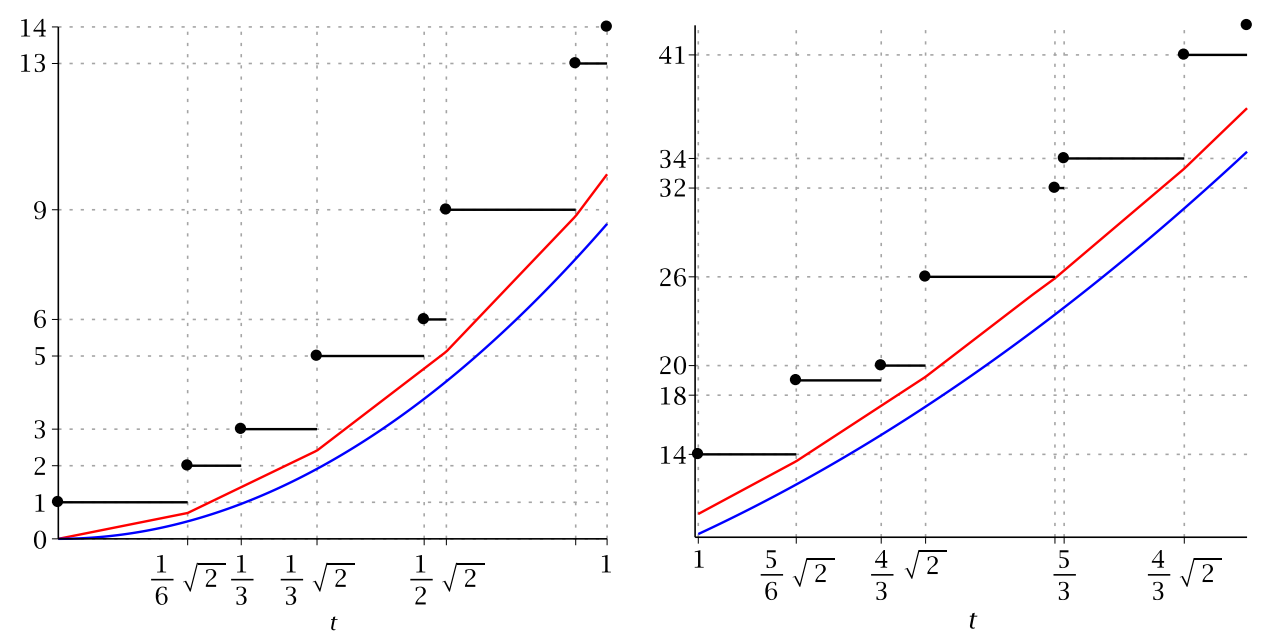

FiguRE 9. $S^{L}\left(\operatorname{tp}_{I}, 1\right)$ where $\mathfrak{p}_{I}$ is the quadrilateral with irrational vertices of Example 2.36, for $L=\{0\}$ (black), $L$ vertical (red), $L=\mathbb{R}^{2}$ (blue). On the left, $t$ varies from 0 to 1 . On the right, $t$ varies from 1 to 2 .

(2) The coefficient functions $E_{r}^{L}(\mathfrak{p}, h)(t)$ are step-polynomials; they belong to the space $\mathcal{Q}_{[\leqslant m+d-r]}^{\Psi}(\mathbb{R})$.

(3) Let $\mathfrak{p}$ be rational and $q \in \mathbb{N}$ is such that $q \mathfrak{p}$ has lattice vertices. Then the coefficient functions $E_{r}^{L}(\mathfrak{p}, h)(t)$ are rational step-polynomials on $\mathbb{R}$; they are periodic functions with period $q$. Thus $E^{L}(\mathfrak{p}, h)(t)$ is a quasi-polynomial.

One can also prove the theorem directly with a proof similar to that of Theorem 2.26, based on Brion's theorem for $\mathfrak{p}$, without embedding $\mathfrak{p}$ in a parametric family. This was done in [5] under the assumption that $\mathfrak{p}$ is rational.

EXAMPLE 2.36 (continuation of Examples 2.8, 2.15). Consider now the quadrilateral $\mathfrak{p}_{I}=\mathfrak{p}\left(b_{I}\right)$ with $b_{I}=[0,0,3 \sqrt{2}, 3]$. Its four vertices are $[0,0],[0,3],\left[-\frac{3}{2}+\frac{3}{2} \sqrt{2}\right.$, $\left.\frac{3}{2}+\frac{3}{2} \sqrt{2}\right]$, and $[3 \sqrt{2}, 0]$.

We specialize the formula which gives $E^{L}\left(\alpha, 1, \tau_{2}\right)(b)$ for $b=t b_{I}$, when $L=V, L$ is the vertical line, $L=\{0\}$, respectively.

(1) First, for $L=V, E^{L}\left(\alpha, 1, \tau_{2}\right)\left(t b_{I}\right)$ is the volume given by

$$
V\left(t \mathfrak{p}_{I}\right)=\frac{9}{4}(1+2 \sqrt{2}) t^{2}
$$

This is a polynomial function of $t$ with real coefficients.

(2) When $L$ is the vertical line, we add the lengths of the vertical segments in $t \mathfrak{p}_{I}$, for $t \geqslant 0 . S^{L}\left(t^{\prime}, 1\right)=E_{2}^{L}(t) t^{2}+E_{1}^{L}(t) t+E_{0}^{L}(t)$ with coefficient functions

$$
\begin{aligned}
& E_{2}^{L}(t)=\frac{9}{4}(1+2 \sqrt{2}), \quad E_{1}^{L}(t)=\frac{3}{2} \\
& E_{0}^{L}(t)=-\frac{1}{2}\{3 \sqrt{2} t\}^{2}+\frac{1}{2}\{3 \sqrt{2} t\}-\left\{-\frac{3}{2} t+\frac{3}{2} \sqrt{2} t\right\}+\left\{-\frac{3}{2} t+\frac{3}{2} \sqrt{2} t\right\}^{2} .
\end{aligned}
$$

This is a semi-quasi-polynomial, but not a quasi-polynomial, because the coefficient function $E_{0}^{L}(t)$ is not periodic but merely bounded.

(3) Finally, we compute the number of integral points $S^{\{0\}}\left(t \mathfrak{p}_{I}, 1\right)$ in $t \mathfrak{p}_{I}$, for $t \geqslant 0$. We have $S^{\{0\}}\left(t_{\mathfrak{p}}, 1\right)=E_{2}(t) t^{2}+E_{1}(t) t+E_{0}(t)$, where the coefficient 
functions $E_{r}(t)$ are step-polynomial functions of $t$,

$$
\begin{aligned}
E_{2}(t)= & \frac{9}{4}(1+2 \sqrt{2}), \\
E_{1}(t)= & \frac{3}{2}+\frac{3}{2}\{3 t\}-\frac{3 \sqrt{2}}{2}\{3 \sqrt{2} t\}-\frac{3}{2}\{3 \sqrt{2} t\}-\frac{3 \sqrt{2}}{2}\{3 t\}+3 \sqrt{2}, \\
E_{0}(t)=1- & \frac{1}{2}\{3 t\}-\{3 \sqrt{2} t\}-\frac{1}{2}\{3 t\}^{2}-\left\{\frac{3}{2} t+\frac{3}{2} \sqrt{2} t\right\}^{2} \\
+ & \left\{\frac{3}{2} t+\frac{3}{2} \sqrt{2} t\right\}\{3 t\}+\{3 \sqrt{2} t\}\left\{\frac{3}{2} t+\frac{3}{2} \sqrt{2} t\right\} .
\end{aligned}
$$

Thus again $S^{\{0\}}\left(\operatorname{pp}_{I}, 1\right)$ is a semi-quasi-polynomial, but not a quasipolynomial. It takes only integral values, and is constant over some intervals with end points of the form $\frac{n}{3}, \frac{n}{3 \sqrt{2}}, \frac{n}{3(1+\sqrt{2})}$ with $n$ an integer.

The graphs of these three semi-quasi-polynomials are displayed in Figure 9.

Let us discuss some qualitative properties of the (semi-)quasi-polynomial function $E^{L}(\mathfrak{p}, h)(t)=\sum_{r=0}^{d+m} E_{r}^{L}(\mathfrak{p}, h)(t) t^{r}$, defined in Theorem 2.35. The coefficient functions $E_{r}^{L}(\mathfrak{p}, h)(t)$ are given by polynomial formulae (with rational coefficients) of functions $\left\{r_{j} t\right\}$, where some $r_{j}$ may be irrational. In particular, $E_{r}^{L}(\mathfrak{p}, h)(t)$ is a bounded function on $\mathbb{R}$. (If the polytope $\mathfrak{p}$ is rational, the coefficients $r_{j}$ are rationals, and $E_{r}^{L}(\mathfrak{p}, h)(t)$ is a periodic function on $\mathbb{R}$ and thus $E^{L}(\mathfrak{p}, h)(t)$ is an ordinary quasi-polynomial function on $\mathbb{R}$.) The individual function $\left\{r_{j} t\right\}$ is right-continuous if $r_{j}>0$, and coincides with the affine linear function $r_{j} t-n$ on the semi-open interval $\left[n / r_{j},(n+1) / r_{j}\left[\right.\right.$. If $r_{j}<0,\left\{r_{j} t\right\}$ is left-continuous. It follows that there is a sequence $0 \leqslant d_{1}<d_{2}<\cdots<d_{i}<d_{i+1}<\cdots$ such that $E_{r}^{L}(\mathfrak{p}, h)(t)$ is given by a polynomial formula on the open interval $] d_{i}, d_{i+1}\left[\right.$. At the points $d_{i}$, the function $E_{r}^{L}(\mathfrak{p}, h)(t)$ may be left-continuous, right-continuous, or $d_{i}$ can be a point of discontinuity. In Section 5.2, examples are given of functions $E^{L}(\mathfrak{p}, h)(t)$ having all types of discontinuity on a discrete set of points (left continuity, right continuity, or discontinuous).

Note, however, that if $0 \in \mathfrak{p}$ (as in Examples 2.34 and 2.36), then the function $E^{L}(\mathfrak{p}, h)(t)$ is right-continuous.

2.5.4. Case of Minkowski linear systems. Let $\mathfrak{p}_{1}, \mathfrak{p}_{2}, \ldots, \mathfrak{p}_{q}$ be semi-rational polytopes in $V$. Then it is well known that there exists a parametric polytope $\mathfrak{p}(b)$ defined by $\alpha_{j}(x) \leqslant b_{j}$ for $1 \leqslant j \leqslant N$, a chamber $\tau \subset \mathbb{R}^{N}$ and values $b^{1}, b^{2}, \ldots, b^{q} \in \bar{\tau}$ of the multi-parameter $b$, such that, for all $t_{i} \geqslant 0$, the Minkowski sum $t_{1} \mathfrak{p}_{1}+t_{2} \mathfrak{p}_{2}+\cdots+t_{q} \mathfrak{p}_{q}$ is the polytope $\mathfrak{p}\left(t_{1} b^{1}+t_{2} b^{2}+\cdots+t_{q} b^{q}\right)$, see for instance [18].

Let us recall how to determine this parametric family. For simplicity, we take the case of two polytopes $\mathfrak{p}_{1}, \mathfrak{p}_{2}$. Then the outer normals $\alpha_{j}$ and the values $b_{j}^{1}, b_{j}^{2}$ are determined in the following way. Without loss of generality, we can assume that $\mathfrak{p}_{1}+\mathfrak{p}_{2}$ is full-dimensional. Let $\left(\alpha_{1}, \ldots, \alpha_{N}\right)$ be the list of outer normal vectors to the facets of $\mathfrak{p}_{1}+\mathfrak{p}_{2}$. Then, for each index $j$, we have $b_{j}^{1}:=\max \left(\left\langle\alpha_{j}, x\right\rangle, x \in \mathfrak{p}_{1}\right)$ and $b_{j}^{2}:=\max \left(\left\langle\alpha_{j}, x\right\rangle, x \in \mathfrak{p}_{2}\right)$. In other words, the facet $\mathfrak{f}\left(\mathfrak{p}_{1}+\mathfrak{p}_{2}, \alpha_{j}\right)$ where $\left\langle\alpha_{j}, x\right\rangle$ reaches its maximum on $\mathfrak{p}_{1}+\mathfrak{p}_{2}$ is the Minkowski sum of the face $\mathfrak{f}\left(\mathfrak{p}_{1}, \alpha_{j}\right)$ of $\mathfrak{p}_{1}$ where $\left\langle\alpha_{j}, x\right\rangle$ reaches its maximum on $\mathfrak{p}_{1}$ and the analogous face $\mathfrak{f}\left(\mathfrak{p}_{2}, \alpha_{j}\right)$ of $\mathfrak{p}_{2}$.

Therefore we obtain the following corollary of Theorem 2.26:

THEOREM 2.37. Let $\mathfrak{p}_{1}, \mathfrak{p}_{2}, \ldots, \mathfrak{p}_{q}$ be (semi-) rational polytopes in $V, L \subseteq V$ a rational subspace and $h(x)$ a weight on $V$. There exists a (semi-)quasi-polynomial function $E\left(t_{1}, t_{2}, \ldots, t_{q}\right)$ on $\mathbb{R}^{q}$ such that for $t_{1} \geqslant 0, \ldots, t_{q} \geqslant 0$, the intermediate weighted sum 
on the polytope $t_{1} \mathfrak{p}_{1}+t_{2} \mathfrak{p}_{2}+\cdots+t_{q} \mathfrak{p}_{q}$ is given by

$$
S^{L}\left(t_{1} \mathfrak{p}_{1}+t_{2} \mathfrak{p}_{2}+\cdots+t_{q} \mathfrak{p}_{q}, h\right)=E\left(t_{1}, t_{2}, \ldots, t_{q}\right) .
$$

An example has already appeared in the introduction as Example 1.3 (Figure 4).

REMARK 2.38. The results in [14] were obtained by writing any "vector dilated polytope" as a Minkowski linear system. ${ }^{(3)}$ In the present article, we take the opposite route, starting with Brion's theorem.

\section{Patched sums And highest polynomial Degree terms of WEIGHTED EHRHART QUASI-POLYNOMIALS}

Let $\mathfrak{p}(b)$ be our parametric polytope. Our next concern is to study the highest polynomial degree terms of the weighted Ehrhart quasi-polynomial

$$
E(\alpha, h, \tau)(b)=E^{\{0\}}(\alpha, h, \tau)(b)=\sum_{x \in \mathfrak{p}(b) \cap \Lambda} h(x)
$$

where $b$ varies in the chamber $\tau$.

Following Barvinok [7], we introduce some particular linear combinations of intermediate weighted sums on polytopes and the analogous linear combinations of intermediate generating functions of a polyhedron.

3.1. Patched Generating FunCtion Associated With a FAMily of SLiCing SubSPACES. Let $\mathcal{L}$ be a finite family of linear subspaces $L \subseteq V$ which is closed under sum. Consider the subset $\bigcup_{L \in \mathcal{L}} L^{\perp}$ of $V^{*}$. As the family $\left\{L^{\perp}: L \in \mathcal{L}\right\}$ is stable under intersection, there exists a unique function $\rho$ on $\mathcal{L}$ such that

$$
\left[\bigcup_{L \in \mathcal{L}} L^{\perp}\right]=\sum_{L \in \mathcal{L}} \rho(L)\left[L^{\perp}\right] .
$$

We call $\rho$ the patching function on $\mathcal{L}$. Let us recall its relationship with the Möbius function of the poset $\mathcal{L}([4$, Lemma 4.1$],[7])$. Let $\hat{\mathcal{L}}$ be the poset obtained by adding a smallest element $\hat{0}$ to $\mathcal{L}$. Denote by $\mu$ its Möbius function.

LEMMA 3.1. The patching function $\rho$ on $\mathcal{L}$ satisfies

$$
\rho(L)=-\mu(\hat{0}, L) .
$$

We consider the following linear combination of intermediate generating functions.

DEFINITION 3.2. The Barvinok patched generating function of a semi-rational polyhedron $\mathfrak{p} \subseteq V$ (with respect to the family $\mathcal{L}$ ) is

$$
S^{\mathcal{L}}(\mathfrak{p})(\xi)=\sum_{L \in \mathcal{L}} \rho(L) S^{L}(\mathfrak{p})(\xi) .
$$

Technically, we will use the shifted version, similar to Definition 3.2:

DEFINITION 3.3

$$
M^{\mathcal{L}}(s, \mathfrak{c})(\xi)=\mathrm{e}^{-\langle\xi, s\rangle} S^{\mathcal{L}}(s+\mathfrak{c})(\xi)=\sum_{L \in \mathcal{L}} \rho(L) M^{L}(s, \mathfrak{c})(\xi) .
$$

We now define a subspace of the space $\mathcal{M}_{\ell}\left(V^{*}\right)$ from Definition 2.1.

Definition 3.4. We introduce the notation $\mathcal{M}_{[\geqslant q]}\left(V^{*}\right)$ for the space of functions $\phi$ in $\mathcal{M}_{\ell}\left(V^{*}\right)$ such that $\phi_{[m]}(\xi)=0$ if $m<q$.

${ }^{(3)}$ Again we note that their results are for the rational case only. 
We now state the approximation theorem of [4] for generating functions of cones.

Theorem 3.5 ([4, Theorem 4.7]). Let $\mathfrak{c}$ be a rational cone. Fix $k, 0 \leqslant k \leqslant d$. Let $\mathcal{L}_{k}$ be a family of subspaces of $V$, closed under sum, such that $\operatorname{lin}(\mathfrak{f}) \in \mathcal{L}_{k}$ for every face $\mathfrak{f}$ of codimension $\leqslant k$ of $\mathfrak{c}$. Let $\rho(L), L \in \mathcal{L}_{k}$, be the patching coefficients of $\mathcal{L}_{k}$, let $S^{\mathcal{L}_{k}}(s+\mathfrak{c})(\xi)$ be the Barvinok patched generating function of Definition 3.2 and let $M^{\mathcal{L}_{k}}(s, \mathfrak{c})(\xi)$ be as in Definition 3.3. Then, for any $s \in V$,

$$
\begin{gathered}
M(s, \mathfrak{c})(\xi)-M^{\mathcal{L}_{k}}(s, \mathfrak{c})(\xi) \in \mathcal{M}_{[\geqslant-d+k+1]}\left(V^{*}\right), \\
S(s+\mathfrak{c})(\xi)-S^{\mathcal{L}_{k}}(s+\mathfrak{c})(\xi) \in \mathcal{M}_{[\geqslant-d+k+1]}\left(V^{*}\right) .
\end{gathered}
$$

We show in the next section that these approximations of generating functions of cones lead to computations of the highest polynomial degree terms of weighted Ehrhart quasi-polynomials of parametric polytopes.

3.2. Highest polynomial Degree terms. Recall the notations of Theorem 2.26. Our key technical result is the following.

THEOREM 3.6. Let $\mathfrak{p}(b) \subset V$ be a parametric polytope. Fix $k, 0 \leqslant k \leqslant d$. Let $h(x)=$ $\frac{\langle\ell, x\rangle^{m}}{m !}$. Let $\tau$ be an admissible chamber for $\mathfrak{p}(b)$.

For each $B \in \mathcal{B}_{\tau}$, let $\mathcal{L}_{k, B}$ be a family of subspaces which contains the faces of $\mathfrak{c}_{B}$ of codimension $\leqslant k$ and is closed under sum. Then for $r \geqslant d+m-k$, the terms of polynomial degree $r$ of the Ehrhart quasi-polynomial $E(\alpha, h, \tau)(b)$ are given by

$$
E_{[r]}(\alpha, h, \tau)(b)=\left.\left(\sum_{B \in \mathcal{B}_{\tau}} M^{\mathcal{L}_{k, B}}\left(s_{B}(b), \mathfrak{c}_{B}\right)_{[m-r]}(\xi) \frac{\left\langle\xi, s_{B}(b)\right\rangle^{r}}{r !}\right)\right|_{\xi=\ell} .
$$

Proof. By Formula (18), the terms of polynomial degree $r$ of the Ehrhart quasipolynomial $E(\alpha, h, \tau)(b)$ are given by

$$
E_{[r]}(\alpha, h, \tau)(b)=\left.\left(\sum_{B \in \mathcal{B}_{\tau}} M\left(s_{B}(b), \mathfrak{c}_{B}\right)_{[m-r]}(\xi) \frac{\left\langle\xi, s_{B}(b)\right\rangle^{r}}{r !}\right)\right|_{\xi=\ell} .
$$

By Theorem 3.5, for $r \geqslant d+m-k$, i.e., $m-r \geqslant-d+k$, we have the equality of the homogeneous components of $\xi$-degree $m-r, M\left(s_{B}(b), \mathfrak{c}_{B}\right)_{[m-r]}(\xi)=$ $M^{\mathcal{L}_{k, B}}\left(s_{B}(b), \mathfrak{c}_{B}\right)_{[m-r]}(\xi)$.

We have an analogous result for the dilation of a single polytope.

Proposition 3.7. Let $\mathfrak{p} \subset V$ be a (semi-)rational polytope. Fix $k, 0 \leqslant k \leqslant d$. Let $h(x)=\frac{\langle\ell, x\rangle^{m}}{m !}$. For each vertex $s$ of $\mathfrak{p}$, let $\mathfrak{c}_{s}$ be the cone of feasible directions of $\mathfrak{p}$ at $s$. For each $s$, let $\mathcal{L}_{k, s}$ be a family of subspaces which contains the faces of $\mathfrak{c}_{s}$ of codimension $\leqslant k$ and is closed under sum. Then for $r \geqslant d+m-k$, the term of polynomial degree $r$ of the Ehrhart (semi-)quasi-polynomial $E(\mathfrak{p}, h)(t)$ is given by

$$
E_{[r]}(\mathfrak{p}, h)(t)=\left.\left(\sum_{s} M^{\mathcal{L}_{k, s}}\left(t s, \mathfrak{c}_{s}\right)_{[m-r]}(\xi) \frac{\langle\xi, s\rangle^{r}}{r !}\right)\right|_{\xi=\ell} t^{r} .
$$

In the case of a single polytope $\mathfrak{p}$, there are two canonical families $\mathcal{L}_{k, s}$ associated with a vertex $s$ of $\mathfrak{p}$. The first one, $\mathcal{L}_{k, s}^{\text {cone-by-cone }}$, is the smallest family which contains the subspaces parallel to the faces of $\mathfrak{p}$ through $s$, and which is closed under sum (this condition is automatic if $\mathfrak{p}$ is simple). This family depends only on the cone $\mathfrak{c}_{s}$. The second family, $\mathcal{L}_{k}^{\text {Barvinok }}$, is the smallest family which contains the subspaces parallel to all the faces of $\mathfrak{p}$ and which is closed under sum. The second family is the one which was originally used by Barvinok in [7]. It depends on the polytope $\mathfrak{p}$ and is the same family at each vertex $s$ of $\mathfrak{p}$. We will return to these two choices in Section 5 
and associate to each of these choices a canonical quasi-polynomial function of the multi-parameter $b$ obtaining our three quasi-polynomials, as promised by the title of our article.

We first give more details on patching functions in two simple cases.

3.3. The PATChing FUnCtion of a Simplicial CONE. Let $\mathfrak{c}$ be a simplicial cone with edge generators $v_{1}, \ldots, v_{d}$. Let us denote here by $\mathcal{L}_{k}(\mathfrak{c})$ the family of subspaces $\operatorname{lin}(\mathfrak{f})$ for faces of codimension $\leqslant k$. This family is closed under sum. We computed the patching function of $\mathcal{L}_{k}(\mathfrak{c})$ in [3]. Let us recall the result.

DeFinition 3.8. Let $\mathcal{J}_{\geqslant(d-k)}^{d}$ be the set of subsets $I \subseteq\{1, \ldots, d\}$ with $|I| \geqslant d-k$.

Recall that we denote by $L_{I}$ the linear space spanned by the vectors $v_{i}, i \in I$. Let $d=\operatorname{dim} V$. Then $I \mapsto L_{I}$ is an isomorphism of posets between $\mathcal{J}_{\geqslant(d-k)}^{d}$ and $\mathcal{L}_{k}(\mathfrak{c})$. We denote by $\rho_{d, k}$ the patching function on $\mathcal{L}_{k}(\mathfrak{c}) .{ }^{(4)}$

Proposition 3.9 ([3, Proposition 29]).

$$
\rho_{d, k}\left(L_{I}\right)=(-1)^{|I|-d+k}\left(\begin{array}{c}
|I|-1 \\
d-k-1
\end{array}\right)
$$

where $\left(\begin{array}{l}a \\ b\end{array}\right)=\frac{a !}{b !(a-b) !}$ is the binomial coefficient.

3.4. The PATChing Function of A Simplex. Let $\mathfrak{p}$ be a $d$-dimensional simplex with vertices $s_{1}, \ldots, s_{d+1}$. We fix $0 \leqslant k \leqslant d$. The faces of $\mathfrak{p}$ of dimension $\geqslant d-k$ are labeled by subsets $I$ of $\{1, \ldots, d+1\}$ of cardinality $|I| \geqslant d-k+1$, the face $\mathfrak{f}_{I}$ being the affine span of the vertices $s_{i}$, for $i \in I$. Let us denote the corresponding linear subspace $\operatorname{lin}\left(\mathfrak{f}_{I}\right)$ by $L_{I}$. Thus $L_{I}$ is the linear span of vectors $s_{i}-s_{j}$ with $i, j \in I$. We consider only the case $k \leqslant d-1$. The family $L_{I}$ is not closed under sum, in general. If $I_{1} \cap I_{2} \neq \varnothing$, we clearly have

$$
L_{I_{1}}+L_{I_{2}}=L_{I_{1} \cup I_{2}} .
$$

On the contrary, if $I_{1} \cap I_{2}=\varnothing$, then the sum $L_{I_{1}} \oplus L_{I_{2}}$ is direct and is not of the form $\operatorname{lin}(\mathfrak{f})$. Therefore, to describe the family $\mathcal{L}_{k}^{\text {Barvinok }}$ for the simplex, we need to consider "subpartitions" $I=\left\{I_{1}, \ldots, I_{m}\right\}$ of $\{1, \ldots, d+1\}$, meaning that $I_{1}, \ldots, I_{m}$ are pairwise disjoint subsets of $\{1, \ldots, d+1\}$. The corresponding subspace is

$$
L_{I}=L_{I_{1}} \oplus \cdots \oplus L_{I_{m}} \subseteq V .
$$

DEFINITION 3.10. We denote by $\Pi_{N, n}$ the poset of all the subpartitions $I=$ $\left\{I_{1}, \ldots, I_{m}\right\}$ of $\{1, \ldots, N\}$, with $m \geqslant 1$ and $\left|I_{j}\right| \geqslant n$, ordered by refinement and set inclusion. ${ }^{(5)}$ (Its least element is the empty subpartition, $\hat{0}=\varnothing$.)

Let $N=d+1, n=d-k+1$. Then the map $I \mapsto L(I)$ is a poset isomorphism of $\Pi_{d+1, d-k+1}$ with the poset $\mathcal{L}_{k}^{\text {Barvinok }}$ associated with the faces of codimension $\leqslant k$ of the $d$-dimensional simplex $\mathfrak{p}$.

We denote by $\sigma_{d, k}(I)$ the patching function on $\mathcal{L}_{k}^{\text {Barvinok }}$. By Lemma 3.1 , it is the opposite of the Möbius function $\mu(\hat{0}, I)$ of the poset $\Pi_{d+1, d-k+1}$.

\footnotetext{
${ }^{(4)}$ In [3], it appears under the name $\lambda_{\text {Möbius }}(I)$.

${ }^{(5)}$ The poset can also be identified with the subposet of those partitions of $\{1, \ldots, N\}$ that have no block sizes in $2,3, \ldots, n-1$. (The singleton blocks represent the elements of $\{1, \ldots, N\}$ not in any of the $I_{j}$.) In this form, the poset appears under the notation $\Pi_{N, n}$ in a paper by A. Björner and L. Lovász [11]; we adopt the same notation.
} 
EXAMPLE 3.11. The patching function on $\mathcal{L}_{2}^{\text {Barvinok }} \simeq \Pi_{4,2}$, associated with the 3dimensional simplex (tetrahedron) and its faces of codimension $\leqslant 2$. The six edges correspond to $\{\{1,2\}\},\{\{1,3\}\}, \ldots$, the four facets to $\{\{1,2,3\}\},\{\{1,2,4\}\}, \ldots$, the simplex itself to $\{\{1,2,3,4\}\}$, and the three planes spanned by the directions of two opposite edges to $\{\{1,2\},\{3,4\}\},\{\{1,3\},\{2,4\}\},\{\{2,3\},\{1,4\}\}$. The Möbius function of $\Pi_{4,2}$ is easy to compute directly. We obtain

$$
\begin{aligned}
\sigma_{3,2}(\{\{i, j\}\}) & =1, \\
\sigma_{3,2}(\{\{i, j, k\}\}) & =-2, \\
\left.\sigma_{3,2}\{\{1,2,3,4\}\}\right) & =6, \\
\sigma_{3,2}(\{\{i, j\},\{k, l\}\}) & =-1 .
\end{aligned}
$$

It turns out that the Möbius function $\mu(\hat{0}, I)$ of the poset $\Pi_{N, n}$ has been computed by A. Björner and L. Lovász in a different context [11, Section 4]. Their result is the following.

Proposition 3.12. Denote by $\mu_{N, n}(I)$ the Möbius function $\mu(\hat{0}, I)$ of the poset $\Pi_{N, n}$. Then,

(1) $\mu_{N, n}\left(I_{1}, \ldots, I_{r}\right)$ depends only on the block-sizes $n_{1}=\left|I_{1}\right|, \ldots, n_{r}=\left|I_{r}\right|$.

$$
\text { Let us write } \mu_{N, n}\left(n_{1}, \ldots, n_{r}\right) \text { for } \mu_{N, n}\left(I_{1}, \ldots, I_{r}\right) \text {. }
$$

(2) $\mu_{N, m}\left(n_{1}, \ldots, n_{r}\right)=\mu_{N, m}\left(n_{1}\right) \ldots \mu_{N, m}\left(n_{r}\right)$.

(3) $\mu_{N, n}(m)=\mu_{m, n}(m)$, for $n \leqslant m \leqslant N$.

(4) Let $\mu_{N}(n)=\mu_{N, n}(N)$ if $N \geqslant n, \mu_{1}(n)=1$ for every $n \geqslant 1$ and $\mu_{N}(n)=0$ for $2 \leqslant N \leqslant n-1$. Consider the generating series

$$
F_{n}(z)=\sum_{N=1}^{\infty} \mu_{N}(n) \frac{z^{N}}{N !} .
$$

Then

$$
\mathrm{e}^{F_{n}(z)}=\sum_{N=0}^{n-1} \frac{z^{N}}{N !} .
$$

Applying Lemma 3.1, we deduce the following computation rules for the patching function $\sigma_{d, k}(I)$.

Proposition 3.13.

(1) $\sigma_{d, k}\left(I_{1}, \ldots, I_{r}\right)$ depends only on the block-sizes $n_{1}=\left|I_{1}\right|, \ldots, n_{r}=\left|I_{r}\right|$.

Let us write $\sigma_{d, k}\left(n_{1}, \ldots, n_{r}\right)$ for $\sigma_{d, k}\left(I_{1}, \ldots, I_{r}\right)$.

(2) $\sigma_{d, k}\left(n_{1}, \ldots, n_{r}\right)=(-1)^{r-1} \sigma_{d, k}\left(n_{1}\right) \ldots \sigma_{d, k}\left(n_{r}\right)$.

(3) For $d-k \leqslant m \leqslant d+1, \frac{\sigma_{d, k}(m)}{m !}$ is the coefficient of degree $m$ of the power series

$$
-\ln \sum_{p=0}^{d-k-1} \frac{z^{p}}{p !}
$$

\section{The COnE-BY-COne PATChed Generating FUnCtion}

Let $\mathfrak{p} \subset V$ be a polytope. Fix $k, 0 \leqslant k \leqslant d$, where $d$ again is the dimension of $\mathfrak{p}$.

If $\mathcal{L}_{k}$ is a family of subspaces which is closed under sum, then it follows from Brion's theorem that

$$
S^{\mathcal{L}_{k}}(\mathfrak{p})(\xi)=\sum_{s \in \mathcal{V}(\mathfrak{p})} S^{\mathcal{L}_{k}}\left(s+\mathfrak{c}_{s}\right)(\xi) .
$$

In particular, the sum $\sum_{s \in \mathcal{V}(\mathfrak{p})} S^{\mathcal{L}_{k}}\left(s+\mathfrak{c}_{s}\right)(\xi)$ is analytic, although each $s$-term is singular at $\xi=0$. The singularities cancel out when we sum over the vertices. 
In contrast, if we take a different family $\mathcal{L}_{k, s}$ for each vertex $s$, the sum over vertices $\sum_{s \in \mathcal{V}(\mathfrak{p})} S^{\mathcal{L}_{k, s}}\left(s+\mathfrak{c}_{s}\right)(\xi)$ need not be analytic.

However, when $\mathfrak{p}$ is a simple polytope (i.e., its cones at vertices $\mathfrak{c}_{s}$ are simplicial), and $\mathcal{L}_{k, s}$ is chosen as the family of subspaces parallel to the faces of $\mathfrak{c}_{s}$ which are of codimension $\leqslant k$, we will show that this sum is actually holomorphic. We will deduce this fact from the computation in [4] of the residues of the generating function of a shifted cone.

Let us give a name and a notation for this sum.

Definition 4.1. Let $\mathfrak{p}$ be a simple polytope. The cone-by-cone patched generating function of $\mathfrak{p}$ is

$$
A^{k}(\mathfrak{p})(\xi)=\sum_{s \in \mathcal{V}(\mathfrak{p})} S^{\mathcal{L}_{k, s}}\left(s+\mathfrak{c}_{s}\right)(\xi)
$$

where, for each vertex $s$ of $\mathfrak{p}, \mathcal{L}_{k, s}$ is the family of subspaces parallel to the faces of $\mathfrak{c}_{s}$ which are of codimension $\leqslant k .^{(6)}$

Proposition 4.2. Let $\mathfrak{p} \subset V$ be a simple polytope. Then $A^{k}(\mathfrak{p})(\xi)$ is analytic near $\xi=0$

Proof. Let $\left(v_{a}\right)$ be a set of pairwise distinct generators of all the edges of $\mathfrak{p}$. By Proposition 3.4 in [4], $S^{L}\left(s+\mathfrak{c}_{s}\right)$ has simple hyperplane poles near $\xi=0$, given by the edges $v_{a}$ of the cone at vertex $s$. It follows that the product $\prod_{a}\left\langle\xi, v_{a}\right\rangle A^{k}(\mathfrak{p})(\xi)$ is analytic. Therefore it is enough to show that for each edge $v_{a}$, the corresponding residue vanishes. In other words, we want to show that

$$
\left.\left(\left\langle\xi, v_{a}\right\rangle A^{k}(\mathfrak{p})(\xi)\right)\right|_{v_{a}^{\perp}}=0 .
$$

Thus, we need only to compute the residues for those vertices $s$ where $v=v_{a}$ is an edge of $c_{s}$. Let $p$ be the projection $V \rightarrow V / \mathbb{R} v$. Let $s$ and $s^{\prime}$ be adjacent vertices of $\mathfrak{p}$ such that $s^{\prime}-s \in \mathbb{R} v$. If we take $v$ to be the edge generator for the cone $\mathfrak{c}_{s}$, then the edge generator for the other cone $\mathfrak{c}_{s^{\prime}}$ is $-v$. Moreover, the projected cones $p\left(s+\mathfrak{c}_{s}\right)$ and $p\left(s^{\prime}+\mathfrak{c}_{s^{\prime}}\right)$ are both equal to the tangent cone at the vertex $p(s)$ of the projected polytope.

Recall that we denote by $\mathcal{J}_{\geqslant d_{0}}^{d}$ the set of subsets $I \subseteq\{1, \ldots, d\}$ of cardinality $|I| \geqslant d_{0}$. For a given vertex $s$ of $\mathfrak{p}$, let $v_{1}, \ldots, v_{d}$ be primitive edge generators at the vertex $s$. For $I \in \mathcal{J}_{\geqslant d_{0}}^{d}$, the subspace generated by $v_{i}$ for $i \in I$ is denoted by $L_{I}^{s}$. Then the Barvinok patched generating function for the cone $s+\mathfrak{c}_{s}$, with respect to the codimension $k=d-d_{0}$, is

$$
S^{\mathcal{L}_{k, s}}\left(s+\mathfrak{c}_{s}\right)(\xi)=\sum_{p \geqslant d_{0}}(-1)^{p-d_{0}}\left(\begin{array}{c}
p-1 \\
d_{0}-1
\end{array}\right) \sum_{I,|I|=p} S^{L_{I}^{s}}\left(s+\mathfrak{c}_{s}\right)(\xi) .
$$

We can label the edges at $s$ and $s^{\prime}$ in such a way that the projections $p\left(L_{I}^{s}\right)$ and $p\left(L_{I}^{s^{\prime}}\right)$ coincide for all $I$. Then by Proposition 3.4 in [4], we conclude that the residues of $S^{\mathcal{L}_{k, s}}\left(s+\mathfrak{c}_{s}\right)(\xi)$ and $S^{\mathcal{L}_{k, s^{\prime}}}\left(s^{\prime}+\mathfrak{c}_{s^{\prime}}\right)(\xi)$ at $v^{\perp}$ cancel out.

REMARK 4.3 (intermediate Todd classes). Let us outline an explanation of the analyticity of $A^{k}(\mathfrak{p})(\xi)$ based on intermediate Todd classes. We will not use this remark in the rest of this article.

When $\mathfrak{p}$ is a simple lattice polytope, we can relate the function $A^{k}(\mathfrak{p})(\xi)$ to some equivariant cohomology classes of the associated toric variety. For simplicity, let us

\footnotetext{
${ }^{(6)}$ This function has appeared in [3], using the notation $A_{\geqslant d-k}(\mathfrak{p})(\xi)$, where $d$ is the dimension of $\mathfrak{p}$.
} 
consider a Delzant polytope $\mathfrak{p}$. Let $G$ be the torus with Lie algebra $\mathfrak{g}=V^{*}$ and weight lattice $\Lambda \subset \mathfrak{g}^{*}=V$.

A smooth toric variety $M$ and $G$-equivariant line bundle $\mathcal{L}$ on $M$ are associated with $\mathfrak{p}$. Let $c_{\mathcal{L}}(\xi)$ be the $G$-equivariant Chern character of $\mathcal{L}$ and let $\operatorname{Todd}^{M}(\xi)$ be the $G$-equivariant Todd class of $M$. The Riemann-Roch theorem relates the set of lattice points of $\mathfrak{p}$ with the integral over $M$ of the product of these two classes:

$$
S(\mathfrak{p})(\xi)=\int_{M} \mathrm{e}^{c_{\mathcal{L}}(\xi)} \operatorname{Todd}^{M}(\xi) .
$$

Brion's formula can be understood as the localization formula applied to this integral. For a generic element $\xi \in \mathfrak{g}$, the vertices of $\mathfrak{p}$ correspond to the fixed points in $M$ under the one-parameter group $\exp (t \xi)$ and the contribution of the fixed point corresponding to the vertex $s$ is precisely $S\left(s+\mathfrak{c}_{s}\right)(\xi)$.

Now, let $c_{1}, \ldots, c_{d}$ be the equivariant Chern classes of the tangent bundle of $M$, so that $\operatorname{Todd}^{M}=\prod_{j=1}^{d} \frac{c_{j}}{1-\mathrm{e}^{-c_{j}}}$. Fix $0 \leqslant k \leqslant d$ and let $d_{0}=d-k$.

Let us introduce the equivariant intermediate Todd class

$$
\operatorname{Todd}_{k}^{M}=\sum_{p \geqslant d_{0}}(-1)^{p-d_{0}}\left(\begin{array}{c}
p-1 \\
d_{0}-1
\end{array}\right) \sum_{I,|I|=p} \prod_{j \in I} \frac{c_{j}}{1-\mathrm{e}^{-c_{j}}} .
$$

We recover the cone-by-cone patched generating function when we replace Todd with $\operatorname{Todd}_{k}$ in (27) and apply the localization formula:

$$
\int_{M} \mathrm{e}^{c_{\mathcal{L}}(\xi)} \operatorname{Todd}_{k}^{M}(\xi)=\sum_{s \in \mathcal{V}(\mathfrak{p})} \sum_{p \geqslant d_{0}}(-1)^{p-d_{0}}\left(\begin{array}{c}
p-1 \\
d_{0}-1
\end{array}\right) \sum_{I,|I|=p} S^{L_{I}}\left(s+\mathfrak{c}_{s}\right)(\xi) .
$$

Now the integral on the left-hand side of (29) depends on $\xi$ analytically.

However, we do not have any geometric interpretation of the quantity computed by the left hand side of (29).

\section{Three Ehrhart QUASI-POLYNOMIALS}

5.1. CASE OF a PARAmetric POLYTOPE. Let $\mathfrak{p}(b) \subset V$ be a parametric polytope, defined by $\alpha: V \rightarrow \mathbb{R}^{N}$, and let $\tau \subset \mathbb{R}^{N}$ be an admissible $\alpha$-chamber. Let $h(x)$ be a weight of degree $m$ on $V$. We fix a codimension $k$. Associated with these data, we have three canonical weighted Ehrhart quasi-polynomials on $\mathbb{R}^{N}$ which have the same terms of polynomial degree $\geqslant d+m-k$.

The first one, $E^{\{0\}}(\alpha, h, \tau)(b)=E(\alpha, h, \tau)(b)$, does not depend on $k$. It is equal, for $b \in \bar{\tau}$, to the ordinary weighted sum $S(\mathfrak{p}(b), h)$. This is the quantity that we want to study.

The second one is associated with the full family of slicing subspaces $\mathcal{L}_{k}^{\text {Barvinok }}$.

DEFINITION 5.1. $E^{k, \text { Barvinok }}(\alpha, h, \tau)(b)$ is the quasi-polynomial such that

$$
E^{k, \text { Barvinok }}(\alpha, h, \tau)(b)=S^{\mathcal{L}_{k}^{\text {Barvinok }}}(\mathfrak{p}(b), h) \text { for } b \in \bar{\tau} .
$$

Our third quasi-polynomial owes its existence to the analyticity of the cone-bycone patched generating function $A^{k}(\mathfrak{p}(b))(\xi)$ of Definition 4.1 for simple polytopes, established in Proposition 4.2. Recall that $\mathfrak{p}(b)$ is simple when $b$ lies in an open chamber. 
Proposition 5.2. Let $\ell \in V^{*}, m \geqslant 0$, and $h(x)=\frac{\langle\ell, x\rangle^{m}}{m !}$. Fix a codimension $k$ and a chamber $\tau$. Then there exists a unique quasi-polynomial $E^{k, \text { cone-by-cone }}(\alpha, h, \tau)(b)$ on $\mathbb{R}^{N}$ such that

$$
E^{k, \text { cone-by-cone }}(\alpha, h, \tau)(b)=A^{k}(\mathfrak{p}(b))_{[m]}(\ell) \quad \text { for } b \in \bar{\tau},
$$

where $A^{k}(\mathfrak{p})_{[m]}(\xi)$ denotes the homogeneous component of degree $m$ with respect to $\xi$.

We extend the definition of $E^{k, \text { cone-by-cone }}(\alpha, h, \tau)(b)$ to arbitrary polynomial functions $h(x)$ on $V$ by decomposition as a sum of powers of linear forms. The piecewise quasi-polynomial $S^{k, \text { cone-by-cone }}(\mathfrak{p}(b), h)$ from the introduction is given on the closure $\bar{\tau}$ of each chamber by $E^{k, \text { cone-by-cone }}(\alpha, h, \tau)(b)$.

Proof. We need only prove that $b \mapsto A^{k}(\mathfrak{p}(b))_{[m]}(\xi)$ is given by a quasi-polynomial function of $b$ (with values in the space of polynomials in $\xi$ ), when $b$ varies in $\tau$. The proof is similar to that of Theorem 2.26 and again relies on [4, Theorem 2.25].

The next theorem follows immediately from Proposition 3.7.

THEOREM 5.3. Let $\mathfrak{p}(b) \subset V$ be a parametric polytope, defined by $\alpha: V \rightarrow \mathbb{R}^{N}$, and let $\tau \subset \mathbb{R}^{N}$ be an $\alpha$-chamber. Fix a codimension $k$. Let $h(x)$ be a polynomial function on $V$ of degree $m$. Then the three quasi-polynomials $E(\alpha, h, \tau)(b), E^{k, \operatorname{Barvinok}}(\alpha, h, \tau)(b)$ and $E^{k, \text { cone-by-cone }}(\alpha, h, \tau)(b)$ have the same terms of polynomial degree $\geqslant d+m-k$.

5.2. Case of a dilated polytope. Polynomial time algorithms. Examples. In this section, we consider just one polytope $\mathfrak{p} \subset V$ and the dilated polytope $t \mathfrak{p}$ for $t$ real $>0$. We fix a weight $h(x)$ on $V$ and a codimension $k$. If $\mathfrak{p}$ is simple, then we have again three canonical quasi-polynomials of the parameter $t$ associated with $\mathfrak{p}$ and $h(x)$, all three of polynomial degree $d+m$. If $\mathfrak{p}$ is not simple, only the first two quasi-polynomials are defined. ${ }^{(7)}$ The first quasi-polynomial is defined by

$$
E(\mathfrak{p}, h)(t)=E^{\{0\}}(\mathfrak{p}, h)(t)=S(t \mathfrak{p}, h) \quad \text { for } t>0 .
$$

The second quasi-polynomial is defined by

$$
E^{k, \text { Barvinok }}(\mathfrak{p}, h)(t)=S^{\mathcal{L}_{k}^{\text {Barvinok }}}(t \mathfrak{p}, h) \quad \text { for } t>0 .
$$

If $\mathfrak{p}$ is simple, the third quasi-polynomial is defined as follows. If $h(x)=\frac{\langle\ell, x\rangle^{m}}{m !}$, then

$$
E^{k, \text { cone-by-cone }}(\mathfrak{p}, h)(t)=A^{k}(t \mathfrak{p})_{[m]}(\ell) \quad \text { for } t>0 .
$$

This definition is again extended to arbitrary polynomial functions $h(x)$ on $V$ by decomposition as a sum of powers of linear forms.

Those three quasi-polynomials have the same terms of polynomial degree $\geqslant d+$ $m-k$.

Furthermore, if $\mathfrak{p}$ is a rational simple polytope, and if $h$ is a power of a rational linear form, $E^{k, \text { Barvinok }}(\mathfrak{p}, h)(t)$ and $E^{k, \text { cone-by-cone }}(\mathfrak{p}, h)(t)$ can be computed in polynomial time when the codimension $k$ is fixed. (We suppress a detailed statement of the algorithm and its complexity.) Similar results on polynomial complexity hold for a more general weight $h(x)$. One can assume that the weight is given as a polynomial in a fixed number $R$ of linear forms, $h(x)=f\left(\left\langle\ell_{1}, x\right\rangle, \ldots,\left\langle\ell_{R}, x\right\rangle\right)$, or has a fixed degree $D$.

${ }^{(7)}$ Of course, we could define $E^{k, \text { cone-by-cone }}(\mathfrak{p}, h)$ as well for non-simple polytopes by taking limits. However, it would no longer be canonical, as it would depend on the path. It is an open question whether a canonical definition is possible, which also should have a toric interpretation (Remark 4.3). 
TABLE 1. The two polynomials $E^{k, \operatorname{Barvinok}}(\mathfrak{p}, 1)(t)$ and $E^{k, \text { cone-by-cone }}(\mathfrak{p}, 1)(t)$ for integer dilations of the lattice simplex of Example 5.4.

\begin{tabular}{ccc}
\hline$k$ & $E^{k, \text { cone-by-cone }}(\mathfrak{p}, 1)(t)$ & $E^{k, \text { Barvinok }}(\mathfrak{p}, 1)(t)$ \\
\hline 0 & $\frac{3}{4} t^{4}$ & $\frac{3}{4} t^{4}$ \\
1 & $\frac{3}{4} t^{4}+2 t^{3}+\frac{7}{24} t^{2}-\frac{5}{5184}$ & $\frac{3}{4} t^{4}+2 t^{3}+\frac{7}{24} t^{2}$ \\
2 & $\frac{3}{4} t^{4}+2 t^{3}+\frac{15}{4} t^{2}+\frac{15}{8} t+\frac{67}{432}$ & $\frac{3}{4} t^{4}+2 t^{3}+\frac{15}{4} t^{2}+\frac{15}{8} t$ \\
3 & $\frac{3}{4} t^{4}+2 t^{3}+\frac{15}{4} t^{2}+\frac{7}{2} t+\frac{389}{432}$ & $\frac{3}{4} t^{4}+2 t^{3}+\frac{15}{4} t^{2}+\frac{7}{2} t$ \\
4 & $\frac{3}{4} t^{4}+2 t^{3}+\frac{15}{4} t^{2}+\frac{7}{2} t+1$ & $\frac{3}{4} t^{4}+2 t^{3}+\frac{15}{4} t^{2}+\frac{7}{2} t+1$ \\
\hline
\end{tabular}

For $E^{k, \text { cone-by-cone }}\left(\mathfrak{p},\langle\ell, x\rangle^{M}\right)(t)$, this result is obtained in [3]. In this article, we used the step-functions $n \mapsto\{\zeta n\}_{q}$ which are defined as $\zeta n \bmod q$ for $\zeta, q, n \in \mathbb{Z}$. However, as noted in [5], the same proof ${ }^{(8)}$ gives the result for real parameters using $t \mapsto q\left\{\frac{\zeta}{q} t\right\}$

For the case of $E^{k, \text { Barvinok }}\left(\mathfrak{p},\langle\ell, x\rangle^{M}\right)(t)$, we apply directly Theorem 28 in [5] where we considered just one intermediate sum $S^{L}(t \mathfrak{p}, h)$. The crucial point is that the codimension of $L$ is bounded by $k$, which is fixed. Here, we need also to compute the patching function. This can be done by computing recursively the Möbius function of the poset $\mathcal{L}_{k}^{\text {Barvinok }}$.

The algorithm for computing $E^{k, \text { cone-by-cone }}\left(\mathfrak{p},\langle\ell, x\rangle^{M}\right)(t)$ is simpler than the original algorithm given by Barvinok in [7], where the subspaces $L$ in the Barvinok family do not necessarily correspond to faces of $\mathfrak{p}$.

We have implemented these algorithms in the case where $\mathfrak{p}$ is a simplex, in Maple. The Maple programs are distributed as part of LattE integrale, version 1.7.2 [1], and also separately via the LattE website. ${ }^{(9)}$ We give below some examples computed with our Maple programs.

In the case of a lattice simplex, when restricted to $t \in \mathbb{N}$, the three quasipolynomials are usual polynomials in $t$. Here is an example.

EXAMPLE 5.4. Let $\mathfrak{p}$ be the 4-dimensional simplex with vertices

$$
[4,6,4,3],[5,7,9,1],[5,7,3,7],[6,8,3,9],[2,1,8,0] .
$$

We use the weight function $h=1$. Table 1 shows the quasi-polynomials $E^{k, \text { Barvinok }}$ $(\mathfrak{p}, 1)(t)$ and $E^{k, \text { cone-by-cone }}(\mathfrak{p}, 1)(t)$, for maximal codimension $k, 0 \leqslant k \leqslant 4$. For $k=0$, both give the volume of the dilated simplex, for $k=4$, both give the exact number of points.

Next, an example of a rational triangle dilated by a real parameter $t$.

EXAMPLE 5.5. Let $\mathfrak{p}$ be the triangle with vertices $[1,1],[1,2],[2,2]$. We use the weight function $h(x)=1$, so we approximate the number of lattice points in the triangle dilated by a real number $t$. We list below the cone-by-cone and the full-Barvinok quasipolynomials, for codimension $k, 0 \leqslant k \leqslant 2$. For $k=0$, both $E^{k, \text { cone-by-cone }}(\mathfrak{p}, 1)(t)$ and $E^{k, \text { Barvinok }}(\mathfrak{p}, 1)(t)$ give the area of the dilated triangle, which is $\frac{t^{2}}{2}$.

\footnotetext{
${ }^{(8)}$ Of course, we can no longer reduce $\zeta$ modulo $q$ in the case of real parameters.

${ }^{(9)}$ The most current versions are available at https ://www. math.ucdavis.edu/ latte/software/ packages/maple/.
} 

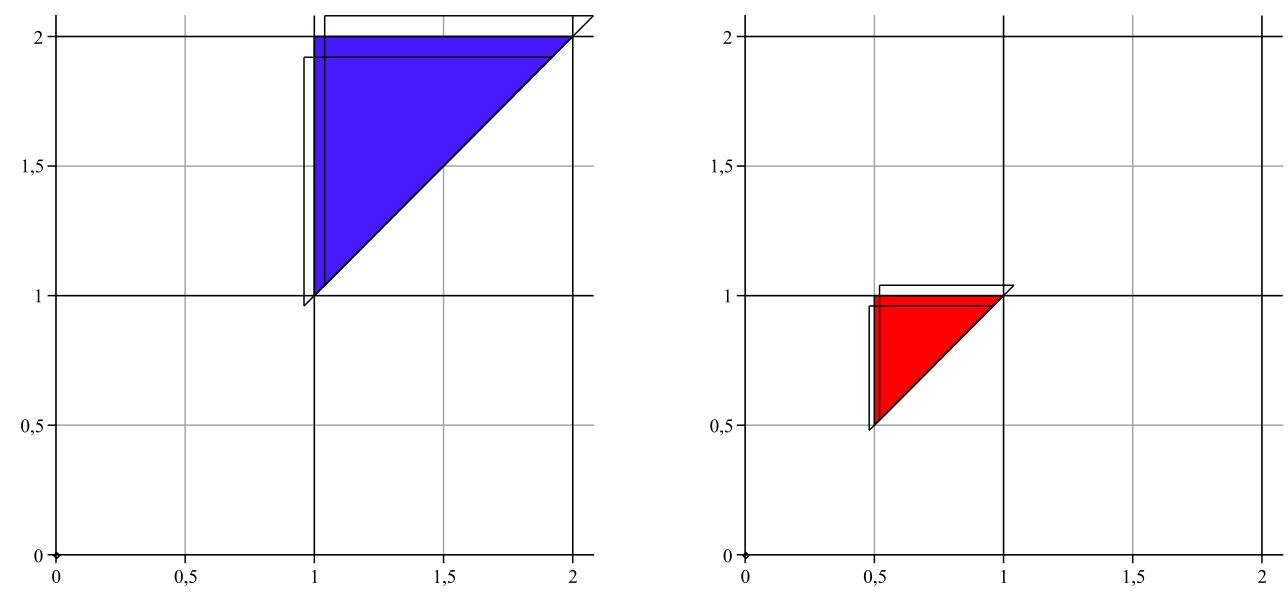

Figure 10. In blue, the triangle with vertices [1, 1], [1,2], and [2,2], dilated by $t=1+\epsilon$ and $t=1-\epsilon$. In red, the same triangle, dilated by $t=\frac{1}{2}$ and $t=\frac{1}{2} \pm \epsilon$.

For $k=1$,

$$
\begin{aligned}
E^{k, \text { cone-by-cone }}(\mathfrak{p}, 1)(t)= & \frac{t^{2}}{2}+\left(\frac{3}{2}-\{-t\}-\{2 t\}\right) t \\
& +\frac{1}{4}-\frac{\{-t\}}{2}-\frac{\{2 t\}}{2}+\frac{\{-t\}^{2}}{2}+\frac{\{2 t\}^{2}}{2}, \\
E^{k, \text { Barvinok }}(\mathfrak{p}, 1)(t)= & \frac{t^{2}}{2}+\left(\frac{3}{2}-\{-t\}-\{2 t\}\right) t-\frac{\{t\}^{2}}{2}+\frac{\{t\}}{2} .
\end{aligned}
$$

For $k=2$, both give the exact number of points,

$$
\text { (32) } \begin{aligned}
\frac{t^{2}}{2}+\left(\frac{3}{2}-\{-t\}-\{2 t\}\right) & t \\
& +\frac{1}{2}\{2 t\}^{2}+\frac{1}{2}\{-t\}^{2}+\{2 t\}\{-t\}-\frac{3}{2}\{-t\}-\frac{3}{2}\{2 t\}+1 .
\end{aligned}
$$

For instance, for $t=\frac{1}{2}, t=\frac{1}{6} \pi=0.52359877 \ldots$, or $t=\frac{1}{2} \sqrt[3]{17 / 10}=0.59674159 \ldots$, the last expression gives 1 , which is indeed the number of lattice points in the triangle with vertices $\left[\frac{1}{2}, \frac{1}{2}\right],\left[\frac{1}{2}, 1\right]$, and $[1,1]$.

On the other hand, for $t=1$, Formula (32) gives 3, which is indeed the number of lattice points in the triangle with vertices $[1,1],[1,2]$, and $[2,2]$, while for $t=1 \pm \epsilon$, with any small $\epsilon$, Formula (32) gives 1 . We leave it as an exercise to the reader to understand the mystery; Figure 10 may help. Figure 11 displays the graphs of the above quasi-polynomials.

In higher dimensions, the quasi-polynomials of a real variable $t$ which arise are too long to display. We will only show some graphs.

EXAMPLE 5.6. Figures 12 and 13 display the graphs of the quasi-polynomials $E^{k, \text { cone-by-cone }}(\mathfrak{p}, 1)(t)$ and $E^{k, \operatorname{Barvinok}}(\mathfrak{p}, 1)(t)$ for the 3-dimensional simplex with vertices

$$
[0,1,1],[4,2,1],[1,1,2],[1,2,4],
$$

for $t \in[1.3,3.9]$. For $k=0$, both quasi-polynomials give the volume $t^{3}$ of the dilated simplex; for $k=3$, they are both equal to $S(t \mathfrak{p}, 1)$ which gives the number of integral 

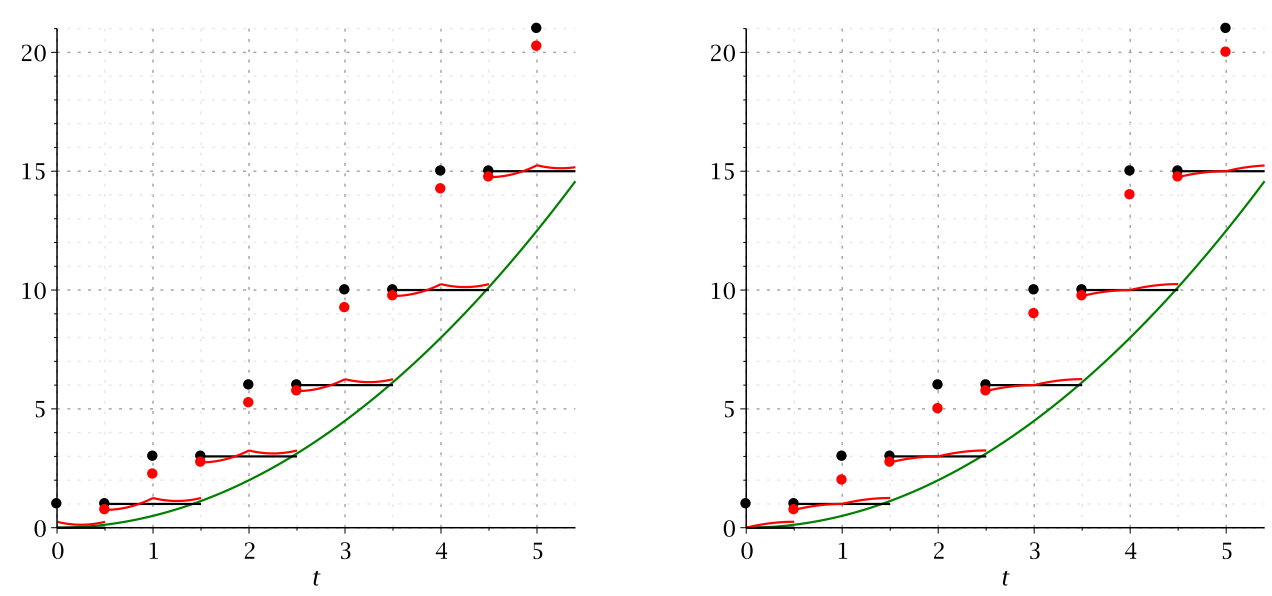

FiguRE 11. Graphs of the quasi-polynomials $E^{k, \text { cone-by-cone }}(\mathfrak{p}, 1)(t)$ (left) and $E^{k, \text { Barvinok }}(\mathfrak{p}, 1)(t)$ (right) for the triangle $\mathfrak{p}$ with vertices $[1,1],[1,2],[2,2]$ and $k=0$ (green), $k=1$ (red), and $k=2$ (black).

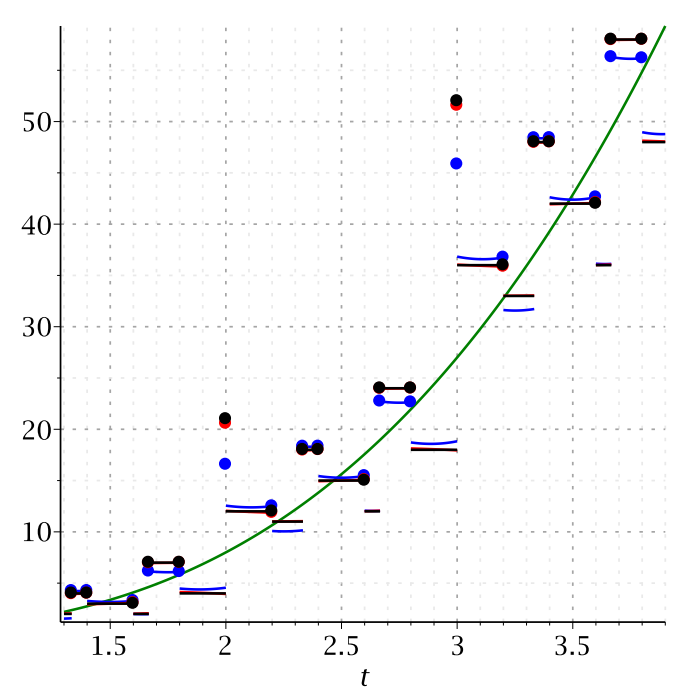

Figure 12. $E^{k, \text { cone-by-cone }}(\mathfrak{p}, 1)(t)$ for the 3 -dimensional simplex with vertices $[0,1,1],[4,2,1],[1,1,2]$, and $[1,2,4]$ from Example 5.6, for $t \in[1.3,3.9]$ and $k=0$ (green), $k=1$ (blue), $k=2$ (red), $k=3$ (black).

points. In this example, we see that the function $t \mapsto S(t \mathfrak{p}, 1)$ has discontinuities on any side (left, right or both).

Acknowledgements. This article is part of a research project which was made possible by several meetings of the authors, at the Centro di Ricerca Matematica Ennio De Giorgi of the Scuola Normale Superiore, Pisa in 2009, in a SQuaRE program at the American Institute of Mathematics, Palo Alto, in July 2009, September 2010, and February 2012, in the Research in Pairs program at Mathematisches Forschungsinstitut Oberwolfach in March/April 2010, and at the Institute for Mathematical Sciences 


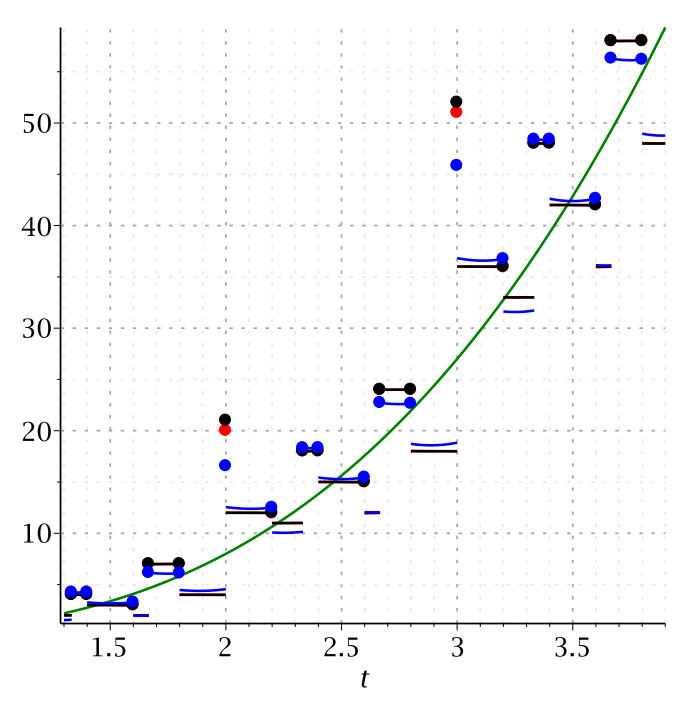

FiguRE 13. $\quad E^{k, \operatorname{Barvinok}}(\mathfrak{p}, 1)(t)$ for the same simplex as in Figure 12.

(IMS) of the National University of Singapore in November/December 2013. The support of all four institutions is gratefully acknowledged. V. Baldoni was partially supported by a PRIN2009 grant. J. De Loera was partially supported by grant DMS0914107 of the National Science Foundation. M. Köppe was partially supported by grant DMS-0914873 of the National Science Foundation.

\section{REFERENCES}

[1] Velleda Baldoni, Nicole Berline, Jesús A. De Loera, Brandon Dutra, Matthias Köppe, Stanislav Moreinis, Gregory Pinto, Michèle Vergne, and Jianqiu Wu, A User's Guide for LattE integrale v1.7.3, 2015, http://www.math.ucdavis.edu/ latte/.

[2] Velleda Baldoni, Nicole Berline, Jesús A. De Loera, Matthias Köppe, and Michèle Vergne, How to Integrate a Polynomial over a Simplex, Math. Comput. 80 (2011), no. 273, 297-325.

[3] Computation of the Highest Coefficients of Weighted Ehrhart Quasi-polynomials of Rational Polyhedra, Found. Comput. Math. 12 (2012), 435-469.

[4] Intermediate Sums on Polyhedra II: Bidegree and Poisson formula, Mathematika 62 (2016), 653-684.

[5] Velleda Baldoni, Nicole Berline, Matthias Köppe, and Michèle Vergne, Intermediate Sums on Polyhedra: Computation and Real Ehrhart Theory, Mathematika 59 (2013), no. 1, 1-22.

[6] Alexander I. Barvinok, Polynomial time algorithm for counting integral points in polyhedra when the dimension is fixed, Math. Oper. Res. 19 (1994), 769-779.

[7] _ Computing the Ehrhart quasi-polynomial of a rational simplex, Math. Comput. 75 (2006), no. 255, 1449-1466.

[8] , Integer Points in Polyhedra, Zürich Lectures in Advanced Mathematics, European Mathematical Society, 2008.

[9] Matthias Beck, Multidimensional Ehrhart Reciprocity, J. Comb. Theory, Ser. A 97 (2002), no. 1, 187-194.

[10] Nicole Berline and Michèle Vergne, Analytic continuation of a parametric polytope and wallcrossing, in Configuration Spaces: Geometry, Combinatorics and Topology, CRM Series, Edizioni della Normale, 2012.

[11] Anders Björner and László Lovász, Linear decision trees, subspace arrangements and Möbius functions, J. Am. Math. Soc. 7 (1994), no. 3, 677-706.

[12] Michel Brion and Michèle Vergne, Residue formulae, vector partition functions and lattice points in rational polytopes, J. Am. Math. Soc. 10 (1997), no. 4, 797-833.

[13] Philippe Clauss and Vincent Loechner, Parametric analysis of polyhedral iteration spaces, Journal of VLSI Signal Processing 19 (1998), no. 2, 179-194. 
[14] Martin Henk and Eva Linke, Lattice points in vector-dilated polytopes, https://arxiv.org/abs/ 1204.6142, 2012.

[15] Allen Knutson and Terence Tao, The honeycomb model of $\mathrm{GL}_{n}(\mathbb{C})$ tensor products. I. Proof of the saturation conjecture, J. Am. Math. Soc. 12 (1999), no. 4, 1055-1090.

[16] Matthias Köppe and Sven Verdoolaege, Computing parametric rational generating functions with a primal Barvinok algorithm, Electron. J. Comb. 15 (2008), article no. R16 (19 pages).

[17] Eva Linke, Rational Ehrhart quasi-polynomials, J. Comb. Theory, Ser. A 118 (2011), no. 7, 1966-1978.

[18] Peter McMullen, Representations of polytopes and polyhedral sets, Geom. Dedicata 2 (1973), 83-99.

[19] Robin Pemantle and Mark C. Wilson, Analytic Combinatorics in Several Variables, Cambridge University Press, 2013.

[20] Sven Verdoolaege, Incremental Loop Transformations and Enumeration of Parametric Sets, Ph.D. thesis, Department of Computer Science, K.U. Leuven (Belgium), 2005.

[21] Sven Verdoolaege, Rachid Seghir, Kristof Beyls, Vincent Loechner, and Maurice Bruynooghe, Counting integer points in parametric polytopes using Barvinok's rational functions, Algorithmica 48 (2007), no. 1, 37-66.

Velleda Baldoni, Dipartimento di Matematica, Università degli studi di Roma "Tor Vergata", Via della ricerca scientifica 1, I-00133, Italy

E-mail : baldoni@mat.uniroma2.it

Nicole Berline, École Polytechnique, Centre de Mathématiques Laurent Schwartz, 91128 Palaiseau Cedex, France

E-mail : nf.berline@gmail.com

Jesús A. De Loera, Department of Mathematics, University of California, Davis, One Shields Avenue, Davis, CA, 95616, USA

E-mail : deloera@math.ucdavis.edu

Matthias Köppe, Department of Mathematics, University of California, Davis, One Shields Avenue, Davis, CA, 95616, USA

E-mail : mkoeppe@math.ucdavis.edu

Michèle Vergne, Université Paris 7 Diderot, Institut Mathématique de Jussieu, Sophie Germain, case 75205, Paris Cedex 13, France E-mail : michele.vergne@imj-prg.fr 